Publication List

Juntendo Medical Journal

2019. 65 (5), 478-500

\title{
Publications from Juntendo University Graduate School of Medicine, 2017 [3/6]
}

\section{Department of Nephrology}

$\langle$ Original Articles〉

1) Wakabayashi K, Io H, Nakata J, Nakamoto H, Sato M, Sasaki Y, Shimizu Y, Horikoshi S, Tomino Y, Suzuki Y: Effects of Cardiac Function with Postoperative Arteriovenous Fistula Blood Flow in Patients with Hemodialysis. Blood Purif, 2017; 44: 24-29.

2) Gohda T, Maruyama S, Kamei N, Yamaguchi S, ShibataT, Murakoshi M, Horikoshi S, Tomino Y, Ohsawa I, Gotoh H, Nojiri S, Suzuki Y: Circulating TNF Receptors 1and 2 Predict Mortality in Patients with End-stage Renal Disease Undergoing Dialysis. Sci Rep, 2017; 7: 43520.

3) Berthoux F, Suzuki H, Mohey H, Maillard N, Mariat C, Novak J, Julian BA: Prognostic Value of Serum Biomarkers of Autoimmunity for Recurrence of IgA Nephropathy after Kidney Transplantation. J Am Soc Nephrol, 2017; 28: 1943-1950.

$\dagger$ 4) Atikemu K, Suzuki Y, Otsuji M, Horikoshi S, Tomino Y: Influence of Estrogen on the Progression of Kindney Injury in Murine IgA Nephropathy. Juntendo Medical Journal, 2017; 63: 178-185.

5) Kanda R, Io H, Nakata J, Makita Y, Sasaki Y, Matsumoto M, Wakabayashi K, Tomino Y, Suzuki Y: Evaluation of Long-Term Combination Therapy with Peritoneal Dialysis and Hemodialysis. Ther Apher Dial, 2017; 21: 180-184.

6) Muto M, Manfroi B, Suzuki H, Joh K, Nagai M, Wakui S, Righini C, Maiguma M, Izui S, Tomino Y, Huard B, Suzuki Y: Toll-like Receptor 9 Stimulation lnduces Aberrant Expression of a Proliferation-lnducing Ligand by Tonsillar Germinal Center B Cell in IgA

An asterisk (*) denotes doctoral works by Japanese students. A dugger $(\dagger)$ denotes doctoral works by non-Japanese students.
Nephropathy. J Am Soc Nephrol, 2017; 28: 1227-1238.

7) Kiryluk K, Li Y, Moldoveanu Z, Suzuki H, Reily C, Hou P, Xie J, Mladkova N, Prakash S, Fischman C, Shapiro S, LeDesma RA, Bradbury D, Ionita-Laza I, Eitner F, Rauen T, Maillard N, Berthoux F, Floege J, Chen N, Zhang H, Scolari F, Wyatt RJ, Julian BA, Gharavi AG, Novak J: GWAS for serum galactose-deficient IgA1 implicates critical genes of the O-glycosylation pathway. PLoS Genet, 2017; 13: e1006609.

8) Yokoro M, Nakayama Y, Yamagishi SI, Ando $\mathrm{R}$, Sugiyama M, Ito S, Yano J, Taguchi K, Kaida Y, Saigusa D, Kimoto M, Abe T, Ueda S, Fukami K: Asymmetric Dimethylarginine Contributes to the Impaired Response to Erythropoietin in CKD-Anemia. J Am Soc Nephrol, 2017; 28: 2670-2680.

* 9) Hara K, Hamada C, Wakabayashi K, Kanda R, Kaneko K, Horikoshi S, Tomino Y, Suzuki Y: Scavenging of reactive oxygen species by astaxanthin inhibits epithelial-mesenchymal transition in high glucose-stimulated mesothelial cells. PLoS One, 2017; 12: e0184332.

10) Sannohe T, Ohnuma T, Takeuchi M, Tani E, Miki Y, Takeda M, Katsuta N, Takebayashi Y, Nakamura T, Nishimon S, Kimoto A, Higashiyama R, Shibata N, Gohda T, Suzuki Y, Yamagishi SI, Tomino Y, Arai H: High doses of antipsychotic polypharmacy are related to an increase in serum levels of pentosidine in patients with schizophrenia. Prog Neuropsychopharmacol Biol Psychiatry, 2017; 76: 4248.

11) Nishimon S, Ohnuma T, Takebayashi $Y$, Katsuta N, Takeda M, Nakamura T, Sannohe T, Higashiyama R, Kimoto A, Shibata N, Gohda T, Suzuki Y, Yamagishi SI, Tomino Y,

This is a reprint of content originally published in Juntendo University HP. 
Arai H: High serum soluble tumor necrosis factor receptor 1 predicts poor treatment response in acute-stage schizophrenia. Prog Neuropsychopharmacol Biol Psychiatry, 2017; 76: 145-154.

12) Jardine MJ, Kasiske B, Adu D, Alrukhaimi M, Ashuntantang GE, Basnet S, Chailimpamontree W, Craig JC, O’Donoghue DJ, Perkovic V, Powe NR, Roberts CJ, Suzuki Y, Tanaka T, Uhlig K: Closing the gap between evidence and practice in chronic kidney disease. Kidney Int Suppl (2011), 2017; 7: 114-121.

13) Sasaki $Y$, Hidaka $T$, Ueno T, Akiba-Takagi M, Oliva Trejo JA, Seki T, Nagai-Hosoe Y, Tanaka E, Horikoshi S, Tomino Y, Suzuki Y, Asanuma K: Sorting Nexin 9 facilitates podocin endocytosis in the injured podocyte. Sci Rep, 2017; 7: 43921.

14) Hosoe-Nagai $Y$, Hidaka $T$, Sonoda A, Sasaki Y, Yamamoto-Nonaka K, Seki T, Asao R, Tanaka E, Trejo JAO, Kodama F, Takagi M, Tada N, Ueno T, Nishinakamura R, Tomino Y, Asanuma K: Re-expression of Sall1 in podocytes protects against adriamycin-induced nephrosis. Lab Invest, 2017; 97: 1306-1320.

15) Taga $Y$, Tanaka K, Hamada C, Kusubata M, Ogawa-Goto K, Hattori S: Hydroxyhomocitrulline Is a Collagen-Specific Carbamylation Mark that Affects Cross-link Formation. Cell Chem Biol, 2017; 24: 1276-1284.

16) Honda D, Ohsawa I, Sato N, Inoshita H, Mano S, Tomino Y, Suzuki Y: Diminished capacity of opsonization and immune complex solubilization, and detection of anti-C1q antibodies in sera from patients with hereditary angioedema. Allergol Int, 2017; 66: 603-609.

17) Pollock C, Zuk A, Anders HJ, Ganji MR, Johnson DW, Kasiske B, Langham RG, Pecoits R, Remuzzi G, Rossert J, Suzuki Y, Tanaka T, Walker R, Yang CW, Bonventre JV: The establishment and validation of novel therapeutic targets to retard progression of chronic kidney disease. Kidney Int Suppl, 2017; 7: 130-137.

18) Murakoshi M, Gohda T, Sonoda Y, Suzuki H, Tomino Y, Horikoshi S, Suzuki Y: Effect of tonsillectomy with steroid pulse therapy on circulating tumor necrosis factor receptors 1 and 2 in IgA nephropathy. Clin Exp Nephrol,
2017; 21: 1068-1074.

19) Kawanishi K, Honda K, Hamada C: Recommendations for pathological diagnosis on biopsy samples from peritoneal dialysis patients. Pleura Peritoneum, 2017; 2: 3-15.

20) Fukami K, Yamagishi S, Kaifu K, Matsui T, Kaida Y, Ueda S, Takeuchi M, Asanuma K, Okuda S: Corrigendum to "Telmisartan inhibits AGE-induced podocyte damage and detachmen" [Microvascular Research 88 (2013) 79-83]. Microvasc Res, 2017; 110: 65.

21) Yamada K, Huang ZQ, Raska M, Reily C, Anderson JC, Suzuki H, Ueda H, Moldoveanu Z, Kiryluk K, Suzuki Y, Wyatt RJ, Tomino Y, Gharavi AG, Weinmann A, Julian BA, Willey CD, Novak J: Inhibition of STAT3 Signaling Reduces IgA1 Autoantigen Production in IgA Nephropathy. Kidney Int Rep, 2017; 2: 11941207.

22) Watanabe H, Goto S, Mori H, Higashi K, Hosomichi K, Aizawa N, Takahashi N, Tsuchida M, Suzuki Y, Yamada T, Horii A, Inoue I, Kurokawa K, Narita I: Comprehensive microbiome analysis of tonsillar crypts in IgA nephropathy. Nephrol Dial Transplant, 2017; 32: 2072-2079.

\section{Department of Obstetrics and Gynecology}

〈Original Articles〉

1) Takeda S, Makino S, Takeda J, Kanayama N, Kubo T, Nakai A, Suzuki S, Seki H, Terui K, Inaba S, Miyata S: Japanese Clinical Practice Guide for Critical Obstetrical Hemorrhage (2017 revision). J Obstet Gynaecol Res, 2017; 43: 1517-1521.

2) Takeda S, Takeda J, Murakami K, Kubo T, Hamada H, Murakami M, Makino S, Itoh H, Ohba T, Naruse K, Tanaka H, Kanayama N, Matsubara S, Sameshima H, Ikeda T: Annual Report of Perinatology Committee, Japan Society of Obstetrics and Gynecology, 2015: Proposal of urgent measures to reduce maternal deaths. J Obstet Gynaecol Res, 2017; 43: 5-7.

3) Katayama M, Hirayama T, Kiyono T, Onuma M, Tani T, Takeda S, Nishimori K, Fukuda T: Immortalized prairie vole-derived fibroblasts (VMF-K4DTs) can be transformed into pluripotent stem cells and provide a usefl tool 
with which to determine optimal reprogramming conditions. J Reprod Dev, 2017; 63: 311-318.

4) Shinohara E, Yoshida K, Sakumoto K, Tada K, Sato S, Kitamura T, Takeda S: Effects of Partners' Attitudes towards Wife's Aspirations on Depression after Childbirth. Open Journal of Depression (OJD), 2017; 6: 79-88.

5) Miyata M, Matsukawa $T$, Suzuki $Y$, Yokoyama K, Takeda S: Psychometric Properties of Japanese Version of the Attitudes towards Fertility and Childbearing Scale (AFCS). BJMMR, 2017; 19: 1-12.

6) Miki T, Nagayoshi M, Takemoto Y, Yamaguchi T, Takeda S, Watanabe S, Tanaka A: Genetic risk of Klinefelter's syndrome in assisted reproductive technology. Reprod Med Biol, 2017; 16: 188-195.

7) Tanaka A, Tanaka I, Yamaguchi T, Miki T, Ikuma S, Nagayoshi M, Takeda S: Development of a new tubal recanalization method using the combination of hysteroscope and laparoscope in the treatment of obstructed fallopian tubes. Gynecol Minim Invasive Ther, 2017; 6: 226-227.

8) Brighton PJ, Maruyama Y, Fishwick K, Vrljicak P, Tewary S, Fujihara R, Muter J, Lucas ES, Yamada T, Woods L, Lucciola R, Hou Lee Y, Takeda S, Ott S, Hemberger M, Quenby S, Brosens JJ: Clearance of senescent decidual cells by uterine natural killer cells drives endometrial remodeling during the window of implantation. Elife, 2017; 6: e31274.

9) Schaap T, Bloemenkamp K, Deneux-Tharaux C, Knight M, Langhoff-Roos J, Sullivan E, van den Akker T; INOSS (Takeda S, Matsubara S, Saito S, Satoh S, et al.): Defining definitions: a Delphi study to develop a core outcome set for conditions of severe maternal morbidity. BJOG, Epub 2017 Aug 24.

10) Miyakoshi K, Otani T, Kondoh E, Makino S, Tanaka M, Takeda S; Perinatal Research Network in Japan: Retrospective multicenter study of leaving the placenta in situ for patients with placenta previa on a cesarean scar. Int J Gynaecol Obstet, Epub 2017 Dec 15.

11) Seto $S$, Itakura $A$, Okagaki R, Suzuki $M$, Ishihara O: An algorithm for the manage- ment of coagulopathy from postpartum hemorrhage, using fibrinogen concentrate as first-line therapy. Int J Obstet Anesth, 2017; 32: 11-16.

12) Matsuoka $R$, Lee $S$, Sato M, Hibiya $R$, Shimanuki Y, Kasai M, Kamiya K, Itakura A, Koike T, Ikeda K: Piezoelectric vibratorstimulated potential and heart rate accelerations detected from the fetus. Int J Pediatr Otorhinolaryngol, 2017; 101: 204-210.

13) Tamura H, Kishi H, Kitade M, Asai-Sato M, Tanaka A, Murakami T, Minegishi T, Sugino $\mathrm{N}$ : Clinical outcomes of infertility treatment for women with adenomyosis in Japan. Reprod Med Biol, 2017; 16: 276-282.

14) Kaneda H, Terao Y, Matsuda Y, Fujino K, Ujihira T, Kusunoki S, Kimura M, Shiraishi A, Kuwatsuru R, Takeda S: The utility and effectiveness of an internal iliac artery balloon occlusion catheter in surgery for large cervical uterine fibroids. Taiwan J Obstet Gynecol, 2017; 56: 502-507.

15) Deguchi M, Yamada H, Sugiura-Ogasawara M, Morikawa M, Fujita D, Miki A, Makino S, Murashima A: Factors associated with adverse pregnancy outcomes in women with antiphospholipid syndrome: A multicenter study. J Reprod Immunol, 2017; 122: 21-27.

16) Makino S, Hirai $C$, Takeda J, Itakura $A$, Takeda S: Relationship between fetal heart rate patterns and a time course for evaluation of fetal well-being: "the 30 minutes rule" for decision of mechanical delivery. Taiwan J Obstet Gynecol, 2017; 56: 788-792.

17) Yamamoto $Y$, Takahashi $K$, Takemoto $Y$, Kobayashi M, Itatani K, Shimizu T, Itakura A, Takeda S: Evaluation of Myocardial Function According to Early Diastolic Intraventricular Pressure Difference in Fetuses. J Am Soc Echocardiogr, 2017; 30: 1130-1137.e1.

18) Nakagawa K, Kwak-Kim J, Kuroda K, Sugiyama R, Yamaguchi K: Immunosuppressive treatment using tacrolimus promotes pregnancy outcome in infertile women with repeated implantation failures. Am J Reprod Immunol, 2017; 78: e12682.

19) Yamaguchi $T$, Ito $M$, Kuroda $K$, Takeda $S$, Tanaka A: The establishment of appropriate methods for egg-activation by human PLCZ1 
RNA injection into human oocyte. Cell Calcium, 2017; 65: 22-30.

20) Nakagawa K, Kuroda K, Sugiyama R, Yamaguchi K: After 12 consecutive miscarriages, a patient received immunosuppressive treatment and delivered an intact baby. Reprod Med Biol, 2017; 16: 297-301.

21) Saito T, Takeda S, Yamagishi $Y$, Kubo R, Kitamura T: Psychotherapy Training on Psychological Mindedness in a Japanese Nurse Population: Effects and Personality Correlates. Healthcare (Basel), 2017; 5. pii: E43.

22) Kusunoki S, Terao Y, Ujihira T, Fujino K, Kaneda H, Kimura M, Ota T, Takeda S: Efficacy of PET/CT to exclude leiomyoma in patients with lesions suspicious for uterine sarcoma on MRI. Taiwan J Obstet Gynecol, 2017; 56: 508-513.

23) Enokizono M, Aida N, Niwa T, Osaka H, Naruto T, Kurosawa K, Ohba C, Suzuki T, Saitsu H, Goto T, Matsumoto N: Neuroimaging findings in Joubert syndrome with C5orf42 mutations: a milder form of molar tooth sign and vermian hypoplasia. J Neurol Sci, 2017; 376: 7-12.

24) Brighton PJ, Maruyama Y, Fishwick K, Vrljicak P, Tewary S, Fujihara R, Muter J, Lucas ES, Yamada T, Woods L, Lucciola R, Hou Lee Y, Takeda S, Ott S, Hemberger M, Quenby S, Brosens JJ: Clearance of senescent decidual cells by uterine natural killer cells in cycling human endometrium. Elife, 2017; 6. pii: e31274.

25) Ozaki R, Kuroda K, Ikemoto Y, Ochiai A, Matsumoto A, Kumakiri J, Kitade M, Itakura A, Muter J, Brosens JJ, Takeda S: Reprogramming of the retinoic acid pathway in decidualizing human endometrial stromal cells. PLoS One, 2017; 12: e0173035.

26) Takeda J, Kumakiri J, Makino S, Itakura A, Takeda S: Laparoscopic removal of uterine vertical compression sutures. Gynecol Minim Invasive Ther, 2017; 6: 73-75.

27) Polettini J, Cobo T, Kacerovsky M, Vinturache AE, Laudanski P, Peelen MJ, Helmer H, Lamont RF, Takeda J, Lapointe J, Torloni MR, Zhong N, Menon R: Biomarkers of spontaneous preterm birth: a systematic review of studies using multiplex analysis. J Perinat Med, 2017; 45: 71-84.

28) Takeda J, Fang X, Olson DM: Pregnant human peripheral leukocyte migration during several late pregnancy clinical conditions: a cross-sectional observational study. BMC Pregnancy Childbirth, 2017; 17: 16.

29) Takeda J, Makino S, Itakura A, Takeda S: Technique of rotational forceps delivery using UTokyo Kielland Forceps. Hypertens Res Pregnancy, 2017; 5: 26-27.

30) Takeda J, Makino S, Itakura A, Takeda S: Technique of forceps delivery using UTokyo Naegele Forceps. Hypertens Res Pregnancy, 2017; 5: 24-25.

31) Murakami K, Yamamoto Y, Fukunaga H, Matsushita M, Hirai C, Makino S, Shimizu T, Itakura A, Takeda S: Predictive markers and prenatal management of isolated fetal complete atrioventricular block: A retrospective review at a single institution. J Obstet Gynaecol Res, Epub 2017 Nov 21.

32) Takahashi M, Takeda J, Ono Y, Nagai T, Seki H, Takeda S: Safety and reliability of forceps delivery based on a 3-dimensional fetal head evaluation a retrospective study. Hypertens Res Pregnancy 2017; 5: 65-72.

33) Ueki N, Kanasaki K, Kanasaki M, Takeda S, Koya D: Catechol-O-Methyltransferase Deficiency Leads to Hypersensitivity of the Pressor Response Against Angiotensin II. Hypertension, 2017; 69: 1156-1164.

34) Kanasaki M, Srivastava SP, Yang F, Xu L, Kudoh S, Kitada M, Ueki N, Kim H, Li J, Takeda S, Kanasaki K, Koya D: Deficiency in catechol-o-methyltransferase is linked to a disruption of glucose homeostasis in mice. Sci Rep, 2017; 7: 7927.

*35) Yoshida E, Terao Y, Hayashi N, Mogushi K, Arakawa A, Tanaka Y, Ito Y, Ohmiya H, Hayashizaki Y, Takeda S, Itoh M, Kawaji H: Promoter-level transcriptome in primary lesion of endometrial cancer identified biomarkers associated with lymph node metastasis. Sci Rep, 2017; 7: 14160.

36) Itamochi H, Oishi T, Oumi N, Takeuchi S, Yoshihara K, Mikami M, Yaegashi N, Terao Y, Takehara K, Ushijima K, Watari H, Aoki D, Kimura T, Nakamura T, Yokoyama Y, 
Kigawa J, Sugiyama T: Whole-genome sequencing revealed novel prognostic biomarkers and promising targets for therapy of ovarian clear cell carcinoma. Br J Cancer, 2017; 117: 717-724.

37) Thornton C, Leaw B, Mallard C, Nair S, Jinnai M, Hagberg H: Cell Death in the Developing Brain after Hypoxia-Ischemia. Front Cell Neurosci, 2017; 11: 248.

38) Kumakiri J, Kikuchi I, Ozaki R, Murakami K, Ikuma S, Kitade M: Comparison of Postoperative Wound Adhesion after Laparoscopic Myomectomy between Barbed and Conventional Absorbable Suture: a Propensity Score-Matched Study. J Minim Invasive Gynecol, 2017; 24: S172.

39) Hutchinson D, McBrien A, Howley L, Yamamoto Y, Sekar P, Motan T, Jain V, Savard W, Hornberger LK: First-Trimester Fetal Echocardiography: Identification of Cardiac Structures for Screening from 6 to 13 weeks' gestational age. J Am Soc Echocardiogr, 2017; 30: 763-772

40) Sugimori $Y$, Ota $T$, Ujihira $T$, Ishiguro $T$, Ogishima D: A phase II randomized study to evaluate the efficacy of aprepitant plus palonosetron for preventing delayed-phase CINV associated with TC therapy in gynaecological cancer. J Obstet Gynaecol Res, 2017; 43: 1454-1459.

41) Yamamoto $Y$, Takahashi K, Takemoto $Y$, Kobayashi M, Itatani K, Shimizu T, Itakura A, Takeda S: Evaluation of Myocardial Function According to Early Diastolic Intraventricular Pressure Difference in Fetuses. J Am Soc Echocardiogr, 2017; 30: 1130-1137.

42) Sakamoto A, Kikuchi I, Shimanuki H, Tejima K, Saito J, Sakai K, Kumakiri J, Kitade M, Takeda S: Initial closed trocar entry for laparoscopic surgery: Technique, umbilical cosmesis, and patient satisfaction. Gynecol Minim Invasive Ther, 2017; 6: 167-172.

$\langle$ Reviews〉

1) Kusunoki S, Huang KG, Magno A, Lee CL: Laparoscopic technique of para-aortic lymph node dissection: a comparison of the different approaches to trans- versus extraperitoneal para-aortic lymphadenectomy. Gynecol Minim Invasive Ther, 2017; 6: 51-57.

\section{Department of Hematology}

〈Original Articles〉

1) Ueda K, Ikeda K, Ikezoe T, Harada-Shirado K, Ogawa K, Hashimoto Y, Sano T, Ohkawara H, Kimura S, Shichishima-Nakamura A, Nakamura Y, Shikama Y, Mori T, Mason PJ, Bessler M, Morishita S, Komatsu N, Shide K, Shimoda K, Koide S, Aoyama K, Oshima M, Iwama A, Takeishi Y: Hmga2 collaborates with JAK2V617F in the development of myeloproliferative neoplasms. Blood Adv, 2017; 1: 1001-1015.

2) Tsukune Y, Sasaki M, Odajima T, Sunami K, Takei T, Moriuchi Y, Iino M, Isoda A, Nakaya A, Muta T, Miyake T, Miyazaki K, Shimizu T, Nakajima K, Igarashi A, Nagafuji K, Kurihara T, Aoyama T, Sugimori H, Komatsu N: Incidence and risk factors of hepatitis B virus reactivation in patients with multiple myeloma in an era with novel agents: a nationwide retrospective study in Japan. Blood Cancer J, 2017; 7: 631.

3) Sunami Y, Araki M, Kan S, Ito A, Hironaka Y, Imai M, Morishita S, Ohsaka A, Komatsu N: Histone Acetyltransferase p300/CREBbinding Protein-associated Factor (PCAF) Is Required for All-trans-retinoic Acid-induced Granulocytic Differentiation in Leukemia Cells. J Biol Chem, 2017; 292: 2815-2829.

4) Sato E, Iriyama N, Tokuhira M, Takaku T, Ishikawa M, Nakazato T, Sugimoto KJ, Fujita H, Fujioka I, Asou N, Komatsu N, Kizaki M, Hatta Y, Kawaguchi T: Introduction of secondgeneration tyrosine kinase inhibitors may reduce the prognostic impact of high-risk patients, according to the European treatment and outcome study (EUTOS) score. Leuk Lymphoma, Epub 2017 Aug 25.

5) Sakurai H, Harada Y, Ogata Y, Kagiyama Y, Shingai N, Doki N, Ohashi K, Kitamura T, Komatsu N, Harada H: Overexpression of RUNX1 short isoform has an important role in the development of myelodysplastic/ myeloproliferative neoplasms. Blood Adv, 2017; 1: 1382-1386.

6) Misawa K, Yasuda H, Araki M, Ochiai T, Morishita S, Nudejima M, Hironaka Y, Shirane S, Edahiro Y, Gotoh A, Ohsaka A, 
Komatsu N: The 2016 WHO diagnostic criteria for polycythemia vera renders an accurate diagnosis to a broader range of patients including masked polycythemia vera: Comparison with the 2008 WHO diagnostic criteria. Am J Hematol, 2017; 92: E128-E130.

7) Komatsu N, Kirito K, Shimoda K, Ishikawa T, Ohishi K, Ohyashiki K, Takahashi N, Okada $\mathrm{H}$, Amagasaki T, Yonezu T, Akashi K: Assessing the safety and efficacy of ruxolitinib in a multicenter, open-label study in Japanese patients with myelofibrosis. Int J Hematol, 2017; 105: 309-317.

8) Kobayashi T, Nannya Y, Ichikawa M, Oritani K, Kanakura Y, Tomita A, Kiyoi H, Kobune M, Kato J, Kawabata H, Shindo M, Torimoto Y, Yonemura Y, Hanaoka N, Nakakuma H, Hasegawa D, Manabe A, Fujishima N, Fujii N, Tanimoto M, Morita Y, Matsuda A, Fujieda A, Katayama N, Ohashi H, Nagai H, Terada Y, Hino M, Sato K, Obara N, Chiba S, Usuki K, Ohta M, Imataki O, Uemura M, Takaku T, Komatsu N, Kitanaka A, Shimoda K, Watanabe K, Tohyama K, Takaori-Kondo A, Harigae H, Arai S, Miyazaki Y, Ozawa K, Kurokawa M; National Research Group on Idiopathic Bone Marrow Failure Syndromes: A nationwide survey of hypoplastic myelodysplastic syndrome (a multicenter retrospective study). Am J Hematol, 2017; 92: 13241332.

9) Kitahara H, Maruyama D, Maeshima AM, Makita S, Miyamoto KI, Fukuhara S, Munakata W, Suzuki T, Kobayashi Y, Tajima K, Terauchi T, Kurihara H, Taniguchi H, Komatsu N, Tobinai K: Prognosis of patients with peripheral $\mathrm{T}$ cell lymphoma who achieve complete response after $\mathrm{CHOP} / \mathrm{CHOP}-$ like chemotherapy without autologous stem cell transplantation as an initial treatment. Ann Hematol, 2017; 96: 411-420.

10) Katayama $Y$, Azechi T, Miyazaki M, Takata T, Sekine M, Matsui H, Hanaki H, Yahara K, Sasano H, Asakura K, Takaku T, Ochiai T, Komatsu N, Chambers HF: Prevalence of Slow-Growth Vancomycin Nonsusceptibility in Methicillin-Resistant Staphylococcus aureus. Antimicrob Agents Chemother, 2017; 61. pii: e00452-17.
11) Harrison CN, Koschmieder S, Foltz L, Guglielmelli P, Flindt T, Koehler M, Mathias J, Komatsu N, Boothroyd RN, Spierer A, Perez Ronco J, Taylor-Stokes G, Waller J, Mesa RA: The impact of myeloproliferative neoplasms (MPNs) on patient quality of life and productivity: results from the international MPN Landmark survey. Ann Hematol, 2017; 96: 1653-1665.

12) Tanaka M, Tashiro H, Omer B, Lapteva N, Ando J, Ngo M, Mehta B, Dotti G, Kinchington PR, Leen AM, Rossig C, Rooney CM: Vaccination Targeting Native Receptors to Enhance the Function and Proliferation of Chimeric Antigen Receptor (CAR) -Modified T Cells. Clin Cancer Res, 2017; 23: 3499-3509.

13) Ikuta K, Hatayama M, Addo L, Toki Y, Sasaki K, Tatsumi Y, Hattori A, Kato A, Kato K, Hayashi H, Suzuki T, Kobune M, Tsutsui M, Gotoh A, Aota Y, Matsuura M, Hamada Y, Tokuda T, Komatsu N, Kohgo Y: Iron overload patients with unknown etiology from national survey in Japan. Int J Hematol, 2017; 105: 353-360.

〈Reviews〉

1) Imai $M$, Araki $M$, Komatsu N: Somatic mutations of calreticulin in myeloproliferative neoplasms. Int J Hematol, 2017; 105: 743-747.

2) Araki M, Komatsu N: Novel molecular mechanism of cellular transformation by a mutant molecular chaperone in myeloproliferative neoplasms. Cancer Sci, 2017; 108: 1907-1912.

\section{Department of Pediatrics and Adolescent Medicine}

$\langle$ Original Articles〉

1) Shiba E, Izawa $K$, Kaitani $A$, Isobe $M$, Maehara A, Uchida K, Maeda K, Nakano N, Ogawa H, Okumura K, Kitamura T, Shimizu T, Kitaura J: Ceramide-CD300f binding inhibits lipopolysaccharide-induced skin inflammation. J Biol Chem, 2017; 292: 9242932.

2) Izawa $K$, Maehara $A$, Isobe $M$, Yasuda $Y$, Urai M, Hoshino Y, Ueno K, Matsukawa T, Takahashi M, Kaitani A, Shiba E, Takamori A, Uchida S, Uchida K, Maeda K, Nakano N, Yamanishi Y, Oki T, Voehringer D, Roers A, Nakae S, Ishikawa J, Kinjo Y, Shimizu T, Ogawa H, Okumura K, Kitamura T, Kitaura J: 
Disrupting ceramide-CD300f interaction prevents septic peritonitis by stimulating neutrophil recruitment. Sci Rep, 2017; 7: 4298.

3) Lo Vecchio A, Liguoro I, Dias JA, Berkley JA, Boey C, Cohen MB, Cruchet S, Salazar-Lindo E, Podder S, Sandhu B, Sherman PM, Shimizu T, Guarino A: Rotavirus immunization: Global coverage and local barriers for implementation. Vaccine, 2017; 35: 1637-1644.

4) Ohtomo Y: Atomoxetine ameliorates nocturnal enuresis with subclinical attention-deficit/ hyperactivity disorder. Pediatr Int, 2017; 59: 181-184.

5) Ohtomo Y: Clonidine may have a beneficial effect on refractory nocturnal enuresis. Pediatr Int, 2017; 59: 711-713.

6) Kamei K, Ishikura K, Sako M, Aya K, Tanaka R, Nozu K, Kaito H, Nakanishi K, Ohtomo Y, Miura K, Takahashi S, Morimoto T, Kubota W, Ito S, Nakamura H, Iijima K; Rituximab for Childhood-Onset Refractory Nephrotic Syndrome (RCRNS) Study Group: Longterm outcome of childhood-onset complicated nephrotic syndrome after a multicenter, doubleblind, randomized, placebo-controlled trial of rituximab. Pediatr Nephrol, 2017; 32: 20712078.

7) Uchida K, Ohtsuka Y, Yoden A, Tajiri H, Kimura $\mathrm{H}$, Isihige $\mathrm{T}$, Yamada $\mathrm{H}$, Arai $\mathrm{K}$, Tomomasa T, Ushijima K, Aomatsu T, Nagata S, Otake K, Matsushita K, Inoue M, Kudo T, Hosoi K, Takeuchi K, Shimizu T: Immunosuppressive medication is not associated with surgical site infection after surgery for intractable ulcerative colitis in children. Intractable Rare Dis Res, 2017; 6: 106-113.

8) Saito Y, Yamanaka G, Shimomura H, Shiraishi K, Nakazawa T, Kato F, Shimizu-Motohashi Y, Sasaki M, Maegaki Y: Reconsideration of the diagnosis and treatment of childhood migraine: A practical review of clinical experience. Brain Dev, 2017; 39: 386-394.

9) Nakazawa $Y$, Kawai T, Arai K, Tamura E, Uchiyama T, Onodera M: Fecal calprotectin rise in chronic granulomatous disease-associated colitis. J Clin Immunol, 2017; 37: 741743.

10) Shimizu H, Arai K, Tang J, Hosoi K, Funayama R: 5-Aminosalicylate intolerance causing exacerbation in pediatric ulcerative colitis. Pediatr Int, 2017; 59: 583-587.

11) Yamamoto $Y$, Takahashi $K$, Takemoto $Y$, Kobayashi M, Itatani K, Shimizu T, Itakura A, Takeda S: Evaluation of myocardial function according to early diastolic intraventricular pressure difference in fetuses. J Am Soc Echocardiogr, 2017; 30: 1130-1137.

12) Iwashima $S$, Uchiyama $H$, Ishikawa $T$, Takigiku K, Takahashi K, Toyono M, Inoue N, Nii M: Measurement of aortic valve coaptation and effective height using echocardiography in patients with ventricular septal defects and aortic valve prolapse. Pediatr Cardiol, 2017; 38: 608-616.

13) Yoshitoshi-Uebayashi EY, Toyoda T, Yasuda K, Kotaka M, Nomoto K, Okita K, Yasuchika K, Okamoto S, Takubo N, Nishikubo T, Soga T, Uemoto S, Osafune K: Modelling ureacycle disorder citrullinemia type 1 with diseasespecific iPSCs. Biochem Biophys Res Commun, 2017; 486: 613-619.

14) Okuno M, Kasahara Y, Onodera M, Takubo N, Okajima M, Suga S, Watanabe N, Suzuki J, Ayabe T, Urakami T, Kawamura T, Kikuchi N, Yokota I, Kikuchi T, Amemiya S, Nakabayashi K, Hayashi K, Hata K, Matsubara Y, Ogata T, Fukami M, Sugihara S: Nucleotide substitutions in CD101, the human homolog of a diabetes susceptibility gene in non-obese diabetic mouse, in patients with type 1 diabetes. J Diabetes Investig, 2017; 8: 286294.

15) Ihara K, Fukano C, Ayabe T, Fukami M, Ogata T, Kawamura T, Urakami T, Kikuchi N, Yokota I, Takemoto K, Mukai T, Nishii A, Kikuchi T, Mori T, Shimura N, Sasaki G, Kizu R, Takubo N, Soneda S, Fujisawa T, Takaya R, Kizaki Z, Kanzaki S, Hanaki K, Matsuura N, Kasahara Y, Kosaka K, Takahashi T, Minamitani K, Matsuo S, Mochizuki H, Kobayashi K, Koike A, Horikawa R, Teno S, Tsubouchi K, Mochizuki T, Igarashi Y, Amemiya S, Sugihara S; Japanese Study Group of Insulin Therapy for Childhood and Adolescent Diabetes (JSGIT): FUT2 non-secretor status is associated with Type 1 diabetes susceptibility in Japanese children. Diabet Med, 2017; 34: $586-589$. 
16) Shoji H, Ikeda N, Kojima C, Kitamura $T$, Suganuma H, Hisata K, Hirayama S, Ueno T, Miida T, Shimizu T: Relationship between copper and lipids and atherogenic indices soon after birth in Japanese preterm infants of 32-35 weeks. J Dev Orig Health Dis, 2017; 8: $256-260$.

17) Shoji H, Taka H, Kaga N, Ikeda N, Kitamura T, Miura Y, Shimizu T: A pilot study of the effect of human breast milk on urinary metabolome analysis in infants. J Pediatr Endocrinol Metab, 2017; 30: 939-946.

18) Fujinaga S, Hirano D, Mizutani A, Sakuraya K, Yamada A, Sakurai S, Shimizu T: Predictors of relapse and long-term outcome in children with steroid-dependent nephrotic syndrome after rituximab treatment. Clin Exp Nephrol, 2017; 21: 671-676.

19) Fujinaga S, Nishizaki N, Ohtomo Y: Initial combination therapy with desmopressin, solifenacin, and alarm for monosymptomatic nocturnal enuresis. Pediatr Int, 2017; 59: 383-384.

20) Fujinaga S, Nishino T: Ten-year treatment with mycophenolate mofetil using therapeutic drug monitoring for childhood-onset lupus nephritis in Japan. Pediatr Nephrol, 2017; 32: 189-190.

21) Fujinaga S, Nishino T: Favorable renal outcome in Japanese children with ANCAassociated pauci-immune glomerulonephritis: impact of urine screening program. Pediatr Nephrol, 2017; 32: 529-530.

22) Fujinaga S, Urushihara Y: Impact of interrupted cyclosporine treatment on the development of chronic nephrotoxicity in children with steroid-dependent nephrotic syndrome. Pediatr Nephrol, 2017; 32: 1469-1470.

23) Fujinaga S, Nishino T, Mizutani A, Sakuraya K, Sakurai S: Transition from lupus podocytopathy to diffuse proliferative lupus nephritis without proteinuria in childhood-onset systemic lupus erythematosus. Clin Nephrol, 2017; 87: 217-219.

24) Fujinaga S, Sakuraya K: Repeated administrations of rituximab along with steroids and Immunosuppressive agents in refractory steroid-resistant nephrotic syndrome. Indian Pediatr, 2017; 54: 49-50.
25) Nomura T, Hisata K, Toyama Y, Sakaguchi K, Igarashi N, Nakao A, Matsunaga N, Komatsu M, Obinata K, Shimizu T: Antimicrobial resistance breakthrough-urinaru tract infections in children under antimicrobial prophylaxis. Hiroshima Journal of Medical Sciences, 2017; 66: 39-44.

26) Hishiki T, Watanabe K, Ida K, Hoshino K, Iehara T, Aoki Y, Kazama T, Kihira K, Takama Y, Taguchi T, Fujimura J, Honda S, Matsumoto K, Mori M, Yano M, Yokoi A, Tanaka Y, Fuji H, Miyazaki O, Yoshimura K, Takimoto T, Hiyama E: The role of pulmonary metastasectomy for hepatoblastoma in children with metastasis at diagnosis: Results from the JPLT-2 study. J Pediatr Surg, 2017; 52: 2051-2055.

27) Suzuki M, Saito N, Minowa K, Kagimoto S, Shimizu T: Validation of severity assessment for acute in children pancreatitis. Pediatr Int, 2017; 59: 1127-1128.

28) Sakaguchi S, Higa T, Suzuki M, Fujimura J, Shimizu T: Prophylactic use of octreotide for asparaginase-induced acute pancreatitis. Int J Hematol, 2017; 106: 266-268.

29) Hoshino E, Hayashi K, Suzuki M, Obatake M, Nakano S, Taura Y, Nio M, Takahashi O: An iPhone application using a novel stool color detection algorithm for biliary atresia screening. Pediatr Surg Int, 2017; 33: 1115-1121.

30) Okumura A, Abe S, Nakazawa M, Ikeno M, Igarashi A, Takasu M, Shimizu T: Attitudes toward driver's licenses for people with epilepsy: 2012 versus 2014. Pediatr Int, 2017; 59: 185-189.

31) Tsumita A, Obinata K, Inoue S, Nakazawa T, Kaneko K, Okumkura A, Shimizu T: Cerebrospinal Fluid Matrix Metalloproteinase-9 Level in Children with Bacterial Meningitis. Juntendo Medical Journal, 2017; 63: 29-33.

32) Nishizaki N, Hirano D, Miyasho T, Obinata K, Shoji H, Shimizu T: Evaluation of urinary IL6 in neonates with septic shock treated with polymyxin B-immobilized fiber column. Pediatr Int, 2017; 59: 1032-1033.

33) Ohkawa N, Shoji H, Ikeda N, Suganuma H, Shimizu T: Relationship between insulin-like growth factor 1, leptin and ghrelin levels and catch-up growth in small for gestational age 
infants of 27-31 weeks during neonatal intensive care unit admission. J Paediatr Child Health, 2017; 53: 62-67.

34) Andica C, Hagiwara A, Nakazawa M, Kumamaru KK, Hori M, Ikeno M, Shimizu T, Aoki S: Synthetic MR Imaging in the diagnosis of bacterial meningitis. Magn Reson Med Sci, 2017; 16: 91-92.

35) Ikuse T, Aoyagi Y, Obayashi N, Jimbo K, Kudo T, Ohtsuka Y, Blanchard TG, Czinn SJ, Shimizu T: Antibiotic resistance of Helicobacter pylori and eradication rate in Japanese pediatric patients. Adv Microbiol, 2017; 7: 241-252.

36) Ikuse $\mathrm{T}$, Kudo $\mathrm{T}$, Obayashi $\mathrm{N}$, Jimbo $\mathrm{K}$, Aoyagi Y, Ohtsuka Y, Blanchard TG, Czinn SJ, Shimizu T: A retrospective analysis of three non-invasive tests for initial diagnosis of Helicobacter pylori infection in children. Adv Microbiol, 2017; 7: 253-264.

37) Sekigawa-Hosozawa M, Tanaka K, Shimizu T, Nakano T, Kitazawa S: A group of very preterm children characterized by atypical gaze patterns. Brain Dev, 2017; 39: 218-224.

38) Inage E, Matsubara T, Honjo A, Baba Y, Yamazaki S, Mori M, Suzuki K, Hara S, Nakazawa T, Obinata K, Ohtsuka Y, Shimizu T: Pediatric admissions due to travel-related asthmatic attacks in Japan: A retrospective case series and case-control study. Allergol Int, 2017; 66: 143-145.

*39) Kurimoto T, Kondo A, Ogino I, Fujimura J, Arakawa A, Arai H, Shimizu T: Effect of O6-methylguanine-DNA methyltransferase methylation in medulloblastoma. Mol Clin Oncol, 2017; 7: 1107-1111.

40) Takagi M, Yoshida M, Nemoto Y, Tamaichi H, Tsuchida R, Seki M, Uryu K, Nishii R, Miyamoto S, Saito M, Hanada R, Kaneko H, Miyano S, Kataoka K, Yoshida K, Ohira M, Hayashi Y, Nakagawara A, Ogawa S, Mizutani S, Takita J: Loss of DNA damage response in neuroblastoma and utility of a PARP inhibitor. J Natl Cancer Inst, 2017; 109: djx062.

41) Takagi M, Ishiwata Y, Aoki Y, Miyamoto S, Hoshino A, Matsumoto K, Nishimura A, Tanaka M, Yanagimachi M, Mitsuiki N, Imai K, Kanegane H, Kajiwara M, Takikawa K,
Mae T, Tomita O, Fujimura J, Yasuhara M, Tomizawa D, Mizutani S, Morio T: HLA haploidentical hematopoietic cell transplantation using clofarabine and busulfan for refractory pediatric hematological malignancy. Int J Hematol, 2017; 105: 686-691.

* 42) Kobayashi M, Takahashi K, Yamada M, Yazaki K, Matsui K, Tanaka N, Shigemitsu S, Akimoto K, Kishiro M, Nakanishi K, Kawasaki S, Nii M, Itatani K, Shimizu T: Assessment of early diastolic intraventricular pressure gradient in the left ventricle among patients with repaired tetralogy of Fallot. Heart Vessel, 2017; 32: 1364-1374.

*43) Honjo A, Nakano N, Yamazaki S, Hara M, Uchida K, Kitaura J, Nishiyama C, Yagita H, Ohtsuka Y, Ogawa H, Okumura K, Shimizu T: Pharmacologic inhibition of Notch signaling suppresses food antigen-induced mucosal mast cell hyperplasia. J Allergy Clin Immunol, 2017; 139: 987-996.e10.

*44) Nakagawa M, Nishizaki N, Endo A, Someya T, Saito Y, Mizutani A, Hara T, Murano Y, Sakuraya K, Hara S, Umino D, Hirano D, Fujinaga S, Ohtomo Y, Shimizu T: Impaired nephrogenesis in neonatal rats with oxygeninduced retinopathy. Pediatr Int, 2017; 59: 704-710.

* 45) Hara T, Ohtomo Y, Endo A, Niijima S, Yasui M, Shimizu T: Evaluation of urinary aquaporin 2 and plasma copeptin as biomarkers of effectiveness of desmopressin acetate for the treatment of monosymptomatic nocturnal enuresis. J Urol, 2017; 198: 921-927.

*46) Hosoi K, Ohtsuka Y, Fujii T, Kudo T, Matsunaga N, Tomomasa T, Tajiri H, Kunisaki R, Ishige T, Yamada H, Arai K, Yoden A, Ushijima K, Aomatsu T, Nagata S, Uchida K, Takeuchi K, Shimizu T: Treatment with infliximab for pediatric Crohn's disease: Nationwide survey of Japan. J Gastroenterol Hepatol, 2017; 32: 114-119.

47) Hosoi K, Arai K, Matsuoka K, Shimizu H, Kamei K, Nakazawa A, Shimizu T, Tang J, Ito $\mathrm{S}$ : Prolonged tacrolimus for pediatric gastrointestinal disorder: Double-edged sword? Pediatr Int, 2017; 59: 588-592.

*48) Yamada M, Takahashi K, Kobayashi M, Yazaki K, Takayasu H, Akimoto K, Kishiro M, 
Inage A, Yoshikawa T, Park IS, Nakanishi K, Kawasaki S, Shimizu T: Mechanisms of left ventricular dysfunction assessed by layerspecific strain analysis in patients with repaired tetralogy of Fallot. Circ J, 2017; 81: 846-854.

49) Kojima C, Shoji H, Ikeda N, Kitamura T, Hisata K, Shimizu T: Association of zinc and copper with clinical parameters in the preterm newborn. Pediatr Int, 2017; 59: 1165-1168.

* 50) Yokokura T, Kamei H, Shibano T, Yamanaka D, Sawada-Yamaguchi R, Hakuno F, Takahashi SI, Shimizu T: The short-stature homeoboxcontaining gene (shox/SHOX) is required for the regulation of cell proliferation and bone differentiation in zebrafish embryo and human mesenchymal stem cells. Front Endocrinol (Lausanne), 2017; 8: 125.

*51) Yaguchi A, Ishibashi T, Terada K, UenoYokohata H, Saito Y, Fujimura J, Shimizu T, Ohki K, Manabe A, Kiyokawa N: EP300ZNF384 fusion gene product up-regulates GATA3 gene expression and induces hematopoietic stem cell gene expression signature in B-cell precursor acute lymphoblastic leukemia cells. Int J Hematol, 2017; 106: 269281.

52) Hirabayashi S, Ohki K, Nakabayashi K, Ichikawa H, Momozawa Y, Okamura K, Yaguchi A, Terada K, Saito Y, Yoshimi A, Ogata-Kawata H, Sakamoto H, Kato M, Fujimura J, Hino M, Kinoshita A, Kakuda H, Kurosawa H, Kato K, Kajiwara R, Moriwaki K, Morimoto T, Nakamura K, Noguchi Y, Osumi T, Sakashita K, Takita J, Yuza Y, Matsuda K, Yoshida T, Matsumoto K, Hata K, Kubo M, Matsubara Y, Fukushima T, Koh K, Manabe A, Ohara A, Kiyokawa N: ZNF384related fusion genes consist of a subgroup with a characteristic immunophenotype in childhood B-cell precursor acute lymphoblastic leukemia. Haematologica, 2017; 102: 118129.

53) Sato M, Shoda T, Shimizu H, Orihara K, Futamura K, Matsuda A, Yamada Y, Irie R, Yoshioka T, Shimizu T, Ohya Y, Nomura I, Matsumoto K, Arai K: Gene expression patterns in distinct endoscopic findings for eosinophilic gastritis in children. J Allergy Clin Immunol Pract, 2017; 5: 1639-1649.e2.

54) Takahashi E, Unoki-Kubota H, Shimizu Y, Okamura T, Iwata W, Kajio H, YamamotoHonda R, Shiga T, Yamashita S, Tobe K, Okumura A, Matsumoto M, Yasuda K, Noda M, Kaburagi Y: Proteomic analysis of serum biomarkers for prediabetes using the LongEvans Agouti rat, a spontaneous animal model of type 2 diabetes mellitus. J Diabetes Investig, 2017; 8: 661-671.

〈Reviews〉

1) Kantake M: Stress, HPA dysfunction, inflammation, and psychomotor disability in preterm birth infants. Advances in medicine and Biology, 2017; 115: 13-22.

\section{Department of Anesthesiology}

〈Original Articles〉

* 1) Yamataka A, Koga H, Ochi T, Imashimizu K, Suzuki K, Kuwatsuru R, Lane G, Nishimura K, Inada E, Suzuki K: Pulmonary lobectomy techniques in infants and children. Pediatr Surg Int, 2017; 33: 483-495.

* 2) Kawagoe I, Hayashida M, Nozumi Y, Banno T, Hirayama S, Suzuki K, Satoh D, Naito Y, Inada $\mathrm{E}$ : A combination of a partial anomalous pulmonary venous connection (papvc) and a lung tumor requiring pulmonary resection. J Cardiothorac Vasc Anesth, 2017; 31: 274-278.

* 3) Yamaguchi K, Kumakura S, Murakami T, Someya A, Inada E, Nagaoka I: Ketamine suppresses the substance $\mathrm{P}$-induced production of IL- 6 and IL- 8 by human U373MG glioblastoma/astrocytoma cells. Int $\mathrm{J} \mathrm{Mol}$ Med, 2017; 39: 687-692.

4) Yamaguchi K, Kumakura S, Murakami T, Someya A, Iseki.M Inada E, Nagaoka I: Antiinflammatory actions of gabapentin and pregabalin on the substance $\mathrm{P}$-induced mitogen-activated protein kinases activation in U373 MG human glioblastoma astrocytoma cells. Mol Med Rep, 2017; 5: 6109-6115.

* 5) Aoyama K, D'Souza R, Inada E, Lapinsky SE, Fowler RA: Measurement properties of comorbidity indices in maternal health research: a systematic review. BMC Pregnancy Childbirth, 2017; 17: 372. 
6) Yamaguchi K, Kumakura S, Someya A, Iseki M, Inada E, Nagaoka I: Anti-inflammatory actions of gabapentin and pregabalin on the substance P-induced mitogen-activated protein kinase activation in U373 MG human glioblastoma astrocytoma cells. Mol Med Rep, 2017; 16: 6109-6115.

7) Yamamoto M, Hayashida M, KakemizuWatanabe M, Ando N, Mukaida H, Kawagoe I, Yusuke S, Inada E: B-type natriuretic peptide and hemoglobin are two major factors significantly associated with baseline cerebral oxygen saturation measured using the INVOS oximeter in patients undergoing offpump coronary artery bypass graft surgery. J Cardiothorac Vasc Anesth, Epub 2017 Apr 13.

* 8) Mukaida H, Hayashida M, Matsushita S, Yamamoto M, Nakamura A, Amano A: Brain natriuretic peptide (BNP) may play a major role in risk stratification based on cerebral oxygen saturation by near-infrared spectroscopy in patients undergoing major cardiovascular surgery. PLoS One, 2017; 12: e0181154.

9) Mizuno J, Mohri S, Yokoyama T, Otsuji M, Arita H, Hanaoka K: Temperature-dependent inotropic and lusitropic indices based on halflogistic time constants for four segmental phases in isovolumic left ventricular pressuretime curve in excised, cross-circulated canine heart. Can J Physiol Pharmacol, 2017; 95: 190-198.

10) Mizuno J, Takahashi T: Evaluation of external pressure to the sacral region in the lithotomy position using the noninvasive pressure distribution measurement system. Ther Clin Risk Manag, 2017; 13: 207-213.

11) Mitaka $C$, Ohnuma $T$, Murayama $T$, Kunimoto K, Nagashima M, Takei T, Iguchi N, Tomita M; JAPAN Investigators: Effects of low-dose atrial natriuretic peptide infusion on cardiac surgery-associated acute kidney injury: A multicenter randomized controlled trial. J Crit Care, 2017; 38: 253-258.

12) Furusawa $H$, Sugiura M, Mitaka $C$, Inase N: Direct hemoperfusion with polymyxin Bimmobilized fibre treatment for acute exacerbation of interstitial pneumonia. Respirology,
2017; 22: 1357-1362.

13) Ishikawa $S$, Tanaka M, Maruyama F, Fukagawa A, Shiota N, Matsumura S, Makita K: Effects of acute kidney injury after liver resection on long-term outcomes. Korean J Anesthesiol, 2017; 70: 527-534.

14) Sato M, Masui K, Sarentonglaga B, Yamaguchi M, Fukumori R, Nagao Y, Sago H, Sumikura H: Influence of maternal remifentanil concentration on fetal-to-maternal ratio in pregnant ewes. J Anesth, 2017; 31: 517522.

15) Kou K, Omae T, Wakabayashi S, Sakuraba S: A case in which a capnometer was useful for diagnosing laryngospasm fllowing administration of sugammadex. JA Clin Rep, 2017; 3: 41.

〈Books〉

1) Kawagoe I, Hayashida M, Satoh D, Inada E: Anesthetic management of a patient with a non-functioning right lung who underwent left lung resection. http://euroanaesthesia2017. esahq.org/wp-content/uploads/2015/10/Abst ract-programme-BY-FIELD-OF-INTEREST. pdf

2) Kawagoe I, Hayashida M, Satoh D, Ishikawa S, Suzuki K, Inada E: Which of Desflurane or Propofol Provides Rapider Emergence From General Anesthesia After Lung Resection? A Prospective, Randomized Study. The ANESTHESIOLOGY ${ }^{\circledR}$ Annual Meeting - American Society of Anesthesiologists, 2017. http:// www.asaabstracts.com/strands/asaabstracts/ abstract.htm? year $=2017 \&$ index $=7 \&$ absnum $=4498$

3) Tanaka M, Satoh D, Komatsu A, Katsuda Y, Kawgaoe I, Inada E: Intra-operative Nitrogen Balance and Blood Glucose During Laparoscopic Colorectal Cancer Surgery. The ANESTHESIOLOGY ${ }^{\circledR}$ Annual Meeting American Society of Anesthesiologists, 2017. http://www.asaabstracts.com/strands/asaab stracts/abstract.htm? year $=2017 \&$ index $=2 \&$ absnum $=4593$

\section{Department of Clinical Laboratory Medicine}

$\langle$ Original Articles〉

1) Suzuki L, Hirayama S, Fukui M, Sasaki M, Hiroi S, Ayaori M, Terai S, Tozuka M, Watada 
$\mathrm{H}$, Miida T: Lipoprotein- $\mathrm{X}$ in cholestatic patients causes xanthomas and promotes foam cell formation in human macrophages. J Clin Lipidol, 2017; 11: 110-118.

2) Nakagawa $T$, Hirayama $S$, Watanabe $T$, Yokomura M, Kohno M, Sato T, Bujo H, Sato A, Murata M, Miida T; JSCC Kanto Study Group: Triglyceride concentrations should be measured after elimination of free glycerol to exclude interindividual variations due to adiposity and fasting status. Clin Chem Lab Med, 2017; 55: e191-e194.

3) Nagasaka H, Komatsu H, Inui A, Nakacho M, Morioka I, Tsukahara H, Kaji S, Hirayama S, Miida T, Kondou H, Ihara K, Yagi M, Kizaki Z, Bessho K, Kodama T, Iijima K, Saheki T, Yorifuji T, Honda A: Circulating tricarboxylic acid cycle metabolite levels in citrindeficient children with metabolic adaptation, with and without sodium pyruvate treatment. Mol Genet Metab, 2017; 120: 207-212.

* 4) Nagao Y, Hirayama S, Kon M, Sasamoto K, Sugihara M, Hirayama A, Isshiki M, Seino U, Miyazaki O, Miida T: Current smokers with hyperlipidemia lack elevated preß1-HDL concentrations. J Clin Lipidol, 2017; 11: 242249.

5) Shoji H, Ikeda N, Kojima C, Kitamura T, Suganuma H, Hisata K, Hirayama S, Ueno T, Miida T, Shimizu T: Relationship between copper and lipids and atherogenic indices soon after birth in Japanese preterm infants of 32-35 weeks. J Dev Orig Health Dis, 2017; 8: $256-260$.

6) Tabe Y, Yamamoto S, Saitoh K, Sekihara K, Monma N, Ikeo K, Mogushi K, Shikami M, Ruvolo VR, Ishizawa J, Hail N Jr, Kazuno S, Igarashi M, Matsushita H, Yamanaka Y, Arai H, Nagaoka I, Miida T, Hayashizaki Y, Konopleva M, Andreeff M: Bone marrow adipocytes facilitate fatty acid oxidation activating AMPK and a transcriptional network supporting survival of acute monocytic leukemia cells. Cancer Res, 2017; 77: 14531464.

7) Miida T, Nishimura K, Hirayama S, Miyamoto Y, Nakamura M, Masuda D, Yamashita S, Ushiyama M, Komori T, Fujita N, Yokoyama S, Teramoto T: Homogeneous assays for LDL-
$\mathrm{C}$ and HDL-C are reliable in both the postprandial and fasting state. J Atheroscler Thromb, 2017; 24: 583-599.

8) Sekihara K, Saitoh K, Yamamoto S, Kikkawa M, Kazuno S, Taka H, Kaga N, Arai H, Miida T, Andreeff M, Konopleva M, Tabe Y: Targeting mantle cell lymphoma metabolism and survival through simultaneous blockade of mTOR and nuclear transporter exportin-1. Oncotarget, 2017; 8: 34552-34564.

* 9) Sasamoto K, Hirayama S, Kon M, Seino U, Ueno T, Nagao Y, Hirayama A, Isshiki M, Idei M, Yano K, Miida T: Changes in apolipoprotein E-containing high-density lipoprotein (HDL) have little impact on HDL-cholesterol measurements using homogeneous assays in normolipidemic and dyslipidemic subjects. Clin Chim Acta, 2017; 470: 56-63.

10) Hirayama $S$, Nagasaka $H$, Nakagawa $S$, Takuwa M, Nakacho M, Yorifuji T, Kondou H, Tsukahara H, Morioka I, Ishida A, Yamato S, Miida T: Growth hormone activates hepatic and cerebral cholesterol metabolism in smallfor-gestational age children without catchup growth. J Clin Lipidol, 2017; 11: 10321042.

11) Ai T, Yuri M, Tabe Y, Kakimoto A, Morishita S, Tsuchiya K, Takamochi K, Kodama Y, Takahashi F, Shigeki M, Horii T, Suzuki K, Takahashi K, Miida T, Ohsaka A: Comparison of the analytical performance between cobas EGFR assay and PCR-clamp method in the detection of EGFR mutations in Japanese non-small cell lung cancer patients. Clin Lab, 2017; 63: 1021-1026.

12) Pang J, Hu M, Lin J, Miida T, Nawawi HM, Park JE, Wu X, Ramli AS, Kim NT, Kwok S, Gonzalez-Santos LE, Su TC, Truong TH, Soran H, Yamashita S, Tomlinson B, Watts GF: An enquiry based on a standardised questionnaire into knowledge, awareness and preferences concerning the care of familial hypercholesterolaemia among primary care physicians in the Asia-Pacific region: the “Ten Countries Study". BMJ Open, 2017; 7: e017817.

13) Hu Z, Murakami T, Tamura H, Reich J, Kuwahara-Arai K, Iba T, Tabe Y, Nagaoka I: Neutrophil extracellular traps induce $\mathrm{IL}-1 \beta$ 
production by macrophages in combination with lipopolysaccharide. Int J Mol Med, 2017; 39: 549-558.

14) Hosoda H, Nakamura $\mathrm{K}, \mathrm{Hu} Z$, Tamura $H$, Reich J, Kuwahara-Arai K, Iba T, Tabe Y, Nagaoaka I: Antimicrobial cathelicidin peptide LL-37 induces NET formation and suppresses the inflammatory response in a mouse septic model. Mol Med Rep, 2017; 16: 5618-5626.

15) Kanayama M, Hayano T, Koebis M, Maeda T, Tabe Y, Horie S, Aiba A: Hyperactive mTOR induces neuroendocrine differentiation in prostate cancer cell with concurrent upregulation of IRF1. Prostate, 2017; 77: 14891498.

16) Shishido Y, Tomoike F, Kimura Y, Kuwata K, Yano T, Fukui K, Fujikawa H, Sekido Y, Murakami-Tonami Y, Kameda T, Shuto S, Abe H: A Covalent G-site Inhibitor for Glutathione S-Transferase Pi (GSTP $\left.{ }_{1-1}\right)$. Chem Commun (Camb), 2017; 53: 1113811141.

17) Tanaka K, Osada H, Murakami-Tonami $Y$, Horio Y, Hida T, Sekido Y: Statin suppresses Hippo pathway-inactivated malignant mesothelioma cells and blocks the YAP/CD44 growth stimulatory axis. Cancer Letters, 2017; 385: 215-224.

18) Nonaka M, Kurebayashi N, Murayama T, Sugihara M, Terawaki K, Shiraishi S, Miyano K, Hosoda H, Kishida S, Kangawa K, Sakurai T, Uezono Y: Therapeutic potential of ghrelin and des-acyl ghrelin against chemotherapyinduced cardiotoxicity. Endocr J, 2017; 64 (Suppl.): S35-S39.

〈Reviews〉

1) Tabe $Y$, Tafuri A, Sekihara $K$, Yang $H$, Konopleva M: Inhibition of mTOR kinase as a therapeutic target for acute myeloid leukemia. Expert Opin Ther Targets, 2017; 21: 705-714.

2) Turker I, Ai T, Itoh H, Horie M: Druginduced fatal arrhythmias: Acquired long QT and Brugada syndromes. Pharmacol Ther, 2017; 176: 48-59.

〈Books〉

1) Tabe Y, Konopleva M: Stem Cell Microenvironments and Beyond. In: Birbrair A, ed.
Advances in Experimental Medicine and Biology. Cham; Springer, 2017; 1041: 19-32.

\section{Department of Aging Biology in Health and Disease}

〈Original Articles〉

1) Yamada T, Kerever A, Yoshimura Y, Suzuki Y, Nonaka R, Higashi K, Toida T, Mercier F, Arikawa-Hirasawa E: Heparan sulfate alterations in extracellular matrix structures and fibroblast growth factor-2 signaling impairment in the aged neurogenic niche. J Neurochem, 2017; 142: 534-544.

2) Sadatsuki R, Kaneko H, Kinoshita M, Futami I, Nonaka R, Culley KL, Otero M, Hada S, Goldring MB, Yamada Y, Kaneko K, ArikawaHirasawa E, Ishijima M: Perlecan is required for the chondrogenic differentiation of synovial mesenchymal cells through regulation of Sox9 gene expression. J Orthop Res, 2017; 35: 837-846.

3) Sato K, Kerever A, Kamagata K, Tsuruta K, Irie R, Tagawa K, Okazawa H, ArikawaHirasawa E, Nitta N, Aoki I, Aoki S: Understanding microstructure of the brain by comparison of neurite orientation dispersion and density imaging (NODDI) with transparent mouse brain. Acta Radiol Open, 2017; 6: 2058460117703816.

\section{Department of Esophageal \& Gastroenterological Surgery}

〈Original Articles〉

* 1) Shibamoto M, Hirata H, Eguchi H, Sawada G, Sakai N, Kajiyama Y, Mimori K: The loss of CASP4 expression is associated with poor prognosis in esophageal squamous cell carcinoma. Oncol Lett, 2017; 13: 1761-1766.

* 2) Iizuka T, Kikuchi D, Hoteya S, Kajiyama Y, Kaise M: Efficacy and safety of endoscopic submucosal dissection for superficial cancer of the cervical esophagus. Endosc Int Open, 2017; 5: E736-E741.

* 3) Mine S, Watanabe M, Okamura A, Imamura Y, Kajiyama Y, Sano T: Superior Thoracic Aperture Size is Significantly Associated with Cervical Anastomotic Leakage After Esophagectomy. World J Surg, 2017; 41: 2598-2604.

* 4) Hattori Y, Tomita N, Yoshino K, Kajiyama Y: 
Significance and Outlook of Preoperative Serum Interleukin- $1 \beta$ and Interleukin -6 as Prognostic Factors in Esophageal Cancer. J Gastroint Dig Syst, 2017; 7: 531.

\section{Department of Coloproctological Surgery}

$\langle$ Original Articles〉

* 1) Ishiyama S, Sakamoto K, Kamiyama H, Niwa K, Sugimoto K, Takahashi M, Kojima Y, Goto M, Okuzawa A, Tomiki Y: Salivary Chromogranin A as a Psychosomatic Stress Marker is suppressed in Laparoscopic Surgery compared with Open Surgery for Colon Cancer. Juntendo Medical Journal, 2017; 63: 88-94.

* 2) Honjo K, Munakata S, Tashiro Y, Salama Y, Shimazu H, Eiamboonsert S, Dhahri D, Ichimura A, Dan T, Miyata T, Takeda K, Sakamoto K, Hattori K, Heissig B: Plasminogen activator inhibitor-1 regulates macrophage-dependent postoperative adhesion by enhancing EGF-HER1 signaling in mice. FASEB J, 2017; 31: 2625-2637.

3) Sugimoto K, Sakamoto K, Ichikawa R, Kure K, Honjo K, Ro H, Takahashi R, Kawano S, Niwa $\mathrm{K}$, Ishiyama S, Kamiyama H, Komiyama H, Takahashi M, Kojima Y, Goto M, Okuzawa A, Ishibiki Y, Tomiki Y: Propensity Score Analysis in the Comparison of Long-Term Outcomes for Locally Advanced Colon Cancer Between Laparoscopic Colectomy and Open Colectomy. Juntendo Medical Journal, 2017; 63: $264-272$.

〈Reviews〉

1) Sakamoto K, Tsukamoto R, Kawano S, Kawai M, Niwa K, Ishiyama S, Sugimoto K, Kamiyama H, Komiyama H, Makoto Takahashi M, Kojima Y, Goto M, Okuzawa A, Tomiki Y: Minimally Invasive Surgery for Colorectal Cancer. Juntendo Medical Journal, 2017; 63: 384-392.

\section{Department of Hepato-Biliary-Pancreatic Surgery}

$\langle$ Original Articles〉

1) Oguro S, Yoshimoto J, Imamura H, Ishizaki $Y$, Kawaski S: Three hundred and sixty-eight consecutive pancreaticoduodenectomies with zero mortality. J Hepatobiliary Pancreat Sci, 2017; 24: 226-234.

2) Ichida $H$, Ishizawa T, Tanaka M, Terasawa
M, Watanabe G, Takeda Y, Matsuki R, Matsumura M, Hata $\mathrm{T}$, Mise $\mathrm{Y}$, Inoue $\mathrm{Y}$, Takahashi Y, Saiura A: Use of inntercostal trocars for laparoscopic resection of subphrenic hepatic tumors. Surg Endosc, 2017; 31: 1280-1286.

* 3) Matsudaira S, Ishizaki Y, Yoshimoto J, Fujiwara N, Kawasaki S: Risk factors for intractable ascites after adult-to-adult living donor liver transplantation using left lobe. Transplantation Direct, 2017; 3: e138.

\section{Department of Gastroenterology and Minimally Invasive Surgery}

〈Original Articles〉

1) Oka S, Sakuramoto S, Chuman M, Aratani K, Wakata M, Miyawaki Y, Gunji H, Sato H, Okamoto K, Yamaguchi S, Koyama I: Successful treatment of refractory complete separation of an esophagojejunal anastomosis after laparoscopic total gastrectomy. BMC Res Notes, 2017; 10: 267.

\section{Department of Breast and Endocrine Surgery}

$\langle$ Original Articles〉

1) Uomori T, Horimoto Y, Mogushi K, Matsuoka J, Saito M: Relationship between alcohol metabolism and chemotherapy-induced emetic events in breast cancer patients. Breast Cancer, 2017; 24: 702-707.

2) Tokuda E, Horimoto Y, Arakawa A, Himuro T, Senuma K, Nakai K, Saito M: Differences in Ki67 expressions between pre- and postneoadjuvant chemotherapy specimens might predict early recurrence of breast cancer. Hum Pathol, 2017; 63: 40-45.

3) Horimoto Y: Some pathological and clinical factors are important to describe association between Ki67 expression and recurrences in breast cancer patients receiving neoadjuvant chemotherapy-reply. Hum Pathol, 2017; 68: 205.

* 4) Igari F, Sato E, Horimoto Y, Takahashi Y, Isomura T, Arakawa A, Kitano S, Saito M: Diagnostic significance of intratumoral CD8+ tumor-infiltrating lymphocytes in medullary carcinoma. Hum Pathol, 2017; 70: 129-138.

5) Mori M, Tokuda E, Horimoto Y, Uomori T, Murakami F, Tanabe M, Shiraishi A, 
Arakawa A, Iijima K, Saito M: Tumor marking for breast-conserving surgery of non-palpable early breast cancer presenting microcalcifications. The Breast, 2017; 32 (Suppl. 1): S119.

6) Tamura $K$, Inoue $K$, Masuda $N$, Takao $S$, Kashiwaba M, Tokuda Y, Iwata H, Yamamoto N, Aogi K, Saeki T, Nakayama T, Sato N, Toyama T, Ishida T, Arioka H, Saito M, Ohno S, Yamauchi H, Yamada K, Watanabe J, Ishiguro H, Fujiwara Y: Randomized phase II study of nab-paclitaxel as first-line chemotherapy in patients with HER2-negative metastatic breast cancer. Cancer Sci, 2017; 108: 987-994.

7) Iwata H, Masuda N, Yamamoto D, Sagara Y, Sato N, Yamamoto Y, Saito M, Fujita T, Oura S, Watanabe J, Tsukabe M, Horiguchi K, Hattori S, Matsuura Y, Kuroi K: Circulating tumor cells as a prognostic marker for efficacy in the randomized phase III JO21095 trial in Japanese patients with HER2-negative metastatic breast cancer. Breast Cancer Res Treat, 2017; 162: 501-510.

8) Yamamoto D, Sato N, Rai Y, Yamamoto Y, Saito M, Iwata H, Masuda N, Oura S, Watanabe J, Hattori S, Matsuura Y, Kuroi K: Efficacy and safety of low-dose capecitabine plus docetaxel versus single-agent docetaxel in patients with anthracycline-pretreated HER2-negative metastatic breast cancer: results from the randomized phase III JO21095 trial. Breast Cancer Res Treat, 2017; 161: 473-482.

9) Ogiya A, Yamazaki K, Horii R, Shien T, Horimoto Y, Masuda N, Inao T, Hosoda M, Ishida N, Osako T, Takahashi M, Endo Y, Miyoshi Y, Yasojima H, Tomioka N, Yamashita H: Erratum to: Post-relapse survival in patients with the early and late distant recurrence in estrogen receptor-positive HER2-negative breast cancer. Breast Cancer, 2017; 24: 642.

10) Ogiya A, Yamazaki K, Horii R, Shien $T$, Horimoto Y, Masuda N, Inao T, Hosoda M, Ishida N, Osako T, Takahashi M, Endo Y, Miyoshi Y, Yasojima H, Tomioka N, Yamashita H: Post-relapse survival in patients with the early and late distant recurrence in estrogen receptor-positive HER2-negative breast cancer. Breast Cancer, 2017; 24: 473482.

11) Sakai $T$, Iwase $T$, Teruya $N$, Kataoka A, Kitagawa D, Nakashima E, Ogiya A, Miyagi Y, Iijima K, Morizono H, Makita M, Gomi N, Oguchi M, Ito Y, Horii R, Akiyama F, Ohno S: Surgical excision without whole breast irradiation for complete resection of ductal carcinoma in situ identified using strict, unified criteria. Am J Surg, 2017; 214: 111-116.

\section{Department of Cardiovascular Surgery}

$\langle$ Original Articles〉

1) Sparks BE, Cavarocchi NC, Hirose H: Extracorporeal membrane oxygenation with multiple-organ failure: Can molecular adsorbent recirculating system therapy improve survival? J Heart Lung Transplant, 2017; 36: 71-76.

2) Dohi S, Yokoyama Y, Yamamoto T, Kuwaki K, Hariya A, Kajimoto K, Yamaoka H, Shimada A, Tsuruta R, Amano A: Push-Up Technique and Anatomical Deployment with the Endurant Stent-Graft System for Severely Angulated Aneurysm Necks. J Endovasc Ther, 2017: 24: 435-439.

3) Azimzadeh N, Baram M, Cavarocchi NC, Hirose H: Prone position: Does it help with acute respiratory distress syndrome (ARDS) requiring extracorporeal membrane oxygenation (ECMO)? OJRD, 2017; 7: 18-24.

4) Unai S, Nguyen M, Tanaka D, Gorbachuk N, Marhefka GD, Hirose H, Cavarocchi NC: Clinical significance of spontaneous echo contrast on extracorporeal membrane oxygenation. Ann Thorac Surg, 2017; 103: 773778.

5) Lamb KM, DiMuzio PJ, Johnson A, Batista P, Moudgill N, McCullough M, Eisenberg JA, Hirose H, Cavarocchi NC: Arterial protocol including prophylactic distal perfusion catheter decreases limb ischemia complications in patients undergoing extracorporeal membrane oxygenation. J Vasc Surg, 2017; 65: 1074-1079.

6) Unai S, Yamane K, Tanaka D, Cook G, Hirose H, Cavarocchi NC, Entwistle JWC 3rd: Quality of life and mid-term survival of patients 
bridged with extracorporeal membrane oxygenation to left ventricular assist device. ASAIO J, 2017; 63: 273-278.

7) Shinohara D, Matsushita S, Yamamoto $T$, Inaba $\mathrm{H}$, Kuwaki K, Shimada A, Amano A: Reduction of c-kit positive cardiac stem cells in patients with atrial fibrillation. J Cardiol, 2017; 69: 712-718.

* 8) Endo D, Kato TS, Iwamura T, Oishi A, Yokoyama Y, Kuwaki K, Inaba H, Amano A: The impact of surgical left atrial appendage amputation/ligation on stroke prevention in patients undergoing off-pump coronary artery bypass grafting. Heart Vessels, 2017; 32: 726-734.

9) Goldhammer JE, Dashiell JM, Davis S, Torjman MC, Hirose H: Use of provider debriefing to improve fast-track extubation rates following cardiac surgery at an academic medical center. Am J Med Qual, Epub 2017 June 19.

10) Mukaida H, Hayashida M, Matsushita S, Yamamoto M, Nakamura A, Amano A: Brain natriuretic peptide (BNP) may play a major role in risk stratification based on cerebral oxygen saturation by near-infrared spectroscopy in patients undergoing major cardiovascular surgery. PLoS One, 2017; 12: e0181154.

* 11) Saito Y, Tambara K, Dohi S, Inaba H, Yamamoto T, Tsuruta R, Hirose H, Amano A: Efficacy of Off-Pump coronary artery bypass grafting using skeletonized bilateral internal thoracic arteries in diabetic patients. Juntendo Medical Journal, 2017; 63: 257-263.

12) Peigh G, Kumar J, Unai S, James DT, Hirose $\mathrm{H}$ : Randomized trial of sternal closure for low risk patients: rigid fixation versus wire closure. Heart Surg Forum, 2017; 20: E164E169.

$\dagger$ 13) Li C, Matsushita S, Li Z, Guan J, Amano A: ckit Positive Cardiac Outgrowth Cells Demonstrate Better Ability for Cardiac Recovery Against Ischemic Myopathy. J Stem Cell Res Ther, 2017; 7. pii: 402.

14) Unai S, Hirose H, Cavarocchi NC: Venoarterial Extracorporeal Membrane Oxygenation: Lower Speed, and You May Be Faster Reply. Ann Thorac Surg, 2017; 104: 725-726.
15) Matsushita S, Minematsu K, Yamamoto $T$, Inaba H, Kuwaki K, Shimada A, Yokoyama Y, Amano A: Factors for c-kit expression in cardiac outgrowth cells and human heart tissue. Int Heart J, 2017; 2; 58: 962-968.

$\langle$ Reviews〉

1) Hirose H, Cavarocchi NC: Neurological complications during veno-venous extracorporeal membrane oxygenation. J Thorac Dis, 2017; 9: 1796-1797.

2) Lamb KM, Hirose H: Vascular complications in extracorporeal membrane oxygenation. Extracorporeal membrane oxygenation. Critical Care Clinic, 2017; 33: 813-824.

3) Hirose H, Pitcher HT, Baram M, Cavarocchi NC: Issues in the intensive care unit for patients with extracorporeal membrane oxygenation. Crit Care Clin, 2017; 33: 855-862.

\section{Department of General Thoracic Surgery}

〈Original Articles〉

1) Nakamura H, Ichikawa $T$, Nakasone $S$, Miyoshi T, Sugano M, Kojima M, Fujii S, Ochiai A, Kuwata T, Aokage K, Suzuki K, Tsuboi M, Ishii G: Abundant tumor promoting stromal cells in lung adenocarcinoma with hypoxic regions. Lung Cancer, Epub 2017 Nov 20.

2) Takamochi K, Nojiri S, Oh S, Matsunaga T, Imashimizu K, Fukui M, Suzuki K: Comparison of digital and traditional thoracic drainage systems for postoperative chest tube management after pulmonary resection: A prospective randomized trial. J Thorac Cardiovasc Surg, Epub 2017 Nov 13.

3) Ueda T, Suzuki K, Matsunaga T, Takamochi K, Oh S: Correction to: Postoperative atrial fibrillation is less frequent in pulmonary segmentectomy compared with lobectomy. Gen Thorac Cardiovasc Surg, Epub 2017 Dec 5.

4) Kohsaka S, Nagano M, Ueno T, Suehara $Y$, Hayashi T, Shimada N, Takahashi K, Suzuki K, Takamochi K, Takahashi F, Mano H: A method of high-throughput functional evaluation of EGFR gene variants of unknown significance in cancer. Sci Transl Med, 2017; 9. pii: eaan6566.

5) Watanabe SI, Nakagawa K, Suzuki K, 
Takamochi K, Ito H, Okami J, Aokage K, Saji H, Yoshioka H, Zenke Y, Aoki T, Tsutani Y, Okada M; Lung Cancer Surgical Study Group (LCSSG) of the Japan Clinical Oncology Group (JCOG): Neoadjuvant and adjuvant therapy for Stage III non-small cell lung cancer. Jpn J Clin Oncol, 2017; 47: 1112-1118.

6) Ueda T, Suzuki K, Matsunaga T, Takamochi $\mathrm{K}$, Oh S: Postoperative atrial fibrillation is less frequent in pulmonary segmentectomy compared with lobectomy. Gen Thorac Cardiovasc Surg, Epub 2017 Nov 13.

7) Hattori A, Matsunaga T, Takamochi K, Oh S, Suzuki K: Radiological classification of multiple lung cancers and the prognostic impact based on the presence of a ground glass opacity component on thin-section computed tomography. Lung Cancer, Epub 2017 Sep 7.

8) Ichikawa T, Saruwatari K, Mimaki S, Sugano M, Aokage K, Kojima M, Hishida T, Fujii S, Yoshida J, Kuwata T, Ochiai A, Suzuki K, Tsuboi M, Goto K, Tsuchihara K, Ishii G: Immunohistochemical and genetic characteristics of lung cancer mimicking organizing pneumonia. Lung Cancer, 2017; 113: 134-139.

9) Matsunaga T, Suzuki K, Takamochi K, Oh S: New simple radiological criteria proposed for multiple primary lung cancers. Jpn J Clin Oncol, 2017; 47: 1073-1077.

10) Jotatsu T, Yagishita S, Tajima K, Takahashi F, Mogushi K, Hidayat M, Wirawan A, Ko R, Kanemaru R, Shimada N, Mitani K, Saito T, Takamochi K, Suzuki K, Kohsaka S, Kojima S, Mukae H, Yatera K, Takahashi K: LSD1/ KDM1 isoform LSD1+8a contributes to neural differentiation in small cell lung cancer. Biochem Biophys Rep, 2017; 9: 86-94.

11) Hattori $A$, Matsunaga $T$, Takamochi $K$, Oh $S$, Suzuki K: Prognostic impact of a ground glass opacity component in the clinical $\mathrm{T}$ classification of non-small cell lung cancer. J Thorac Cardiovasc Surg, 2017; 154: 21022110.e1.

12) Honma Y, Togo S, Shimizu K, Tulafu M, Hayashi T, Uekusa T, Tominaga S, Kido K, Fujimoto Y, Nanba Y, Takamochi K, Oh S, Suzuki K, Takahashi K: Expression of thymidylate synthase predicts clinical outcomes of S-1-based chemotherapy in squamous cell lung cancer. Oncol Lett, 2017; 14: 3319-3326.

13) Takamochi K, Oh S, Matsunaga T, Suzuki K: Prognostic impacts of EGFR mutation status and subtype in patients with surgically resected lung adenocarcinoma. J Thorac Cardiovasc Surg, 2017; 154: 1768-1774.e1.

14) Taniguchi D, Oji $Y$, Ueno $Y$, Hirayama S, Fukui M, Miyamoto N, Yamashiro K, Tanaka R, Suzuki K, Hattori N: Limb-Shaking Transient Ischemic Attack Induced by Middle Cerebral Artery Dissection after Lung Surgery. J Stroke Cerebrovasc Dis, 2017; 26: e197-e198.

15) Hara K, Saito T, Hayashi T, Mitani K, Takamochi K, Oh S, Suzuki K, Yao T: Inverse correlation between galectin- 4 and TTF- 1 in lung adenocarcinoma. Virchows Arch, 2017; 471: 375-382.

16) Takamochi K, Takahashi F, Suehara Y, Sato E, Kohsaka S, Hayashi T, Kitano S, Uneno T, Kojima S, Takeuchi K, Mano H, Suzuki K: DNA mismatch repair deficiency in surgically resected lung adenocarcinoma: Microsatellite instability analysis using the Promega panel. Lung Cancer, 2017; 110: 26-31.

17) Hattori A, Matsunaga $T$, Takamochi $K$, Oh $S$, Suzuki K: Indications for sublobar resection of clinical stage IA radiologic pure-solid lung adenocarcinoma. J Thorac Cardiovasc Surg, 2017; 154: 1100-1108.

18) Agatsuma H, Yoshida K, Yoshino I, Okumura M, Higashiyama M, Suzuki K, Tsuchida M, Usuda J, Niwa H: Video-Assisted Thoracic Surgery Thymectomy Versus Sternotomy Thymectomy in Patients with Thymoma. Ann Thorac Surg, 2017; 104: 1047-1053.

19) Suzuki K: Whack-a-mole strategy for multifocal ground glass opacities of the lung. J Thorac Dis, 2017; 9 (Suppl 3): S201-S207.

20) Hattori A, Matsunaga T, Takamochi K, Oh S, Suzuki K: Importance of Ground Glass Opacity Component in Clinical Stage IA Radiologic Invasive Lung Cancer. Ann Thorac Surg, 2017; 104: 313-320.

21) Togo S, Katagiri N, Namba Y, Tulafu M, Nagahama K, Kadoya K, Takamochi K, Oh S, Suzuki K, Sakurai F, Mizuguchi H, Urata Y, Takahashi K: Sensitive detection of viable circulating tumor cells using a novel 
conditionally telomerase-selective replicating adenovirus in non-small cell lung cancer patients. Oncotarget, 2017; 8: 34884-34895.

22) Fukui M, Suzuki K, Matsunaga $T$, Oh S, Takamochi K: Surgical intervention for ground glass dominant lesions: observation or outright resection? Jpn J Clin Oncol, 2017; 47: 749-754.

23) Takamochi K, Mogushi K, Kawaji H, Imashimizu K, Fukui M, Oh S, Itoh M, Hayashizaki Y, Ko W, Akeboshi M, Suzuki K: Correlation of EGFR or KRAS mutation status with $18 \mathrm{~F}-\mathrm{FDG}$ uptake on PET-CT scan in lung adenocarcinoma. PLoS One, 2017; 12: e0175622.

24) Hattori A, Matsunaga T, Takamochi K, Oh S, Suzuki K: Surgical resection for clinicalStage I radiological pure-solid lung cancer that met the current high risk criteria. Jpn J Clin Oncol, 2017; 47: 630-638.

25) Fukui M, Takamochi K, Oh S, Matsunaga T, Suzuki K, Ando K, Suzuki K: Utility of Objective Chest Tube Management After Pulmonary Resection Using a Digital Drainage System. Ann Thorac Surg, 2017; 104: 275-283.

26) Fukui M, Takamochi K, Oh S, Matsunaga T, Suzuki K, Ando K, Suzuki K: Study on Perioperative Administration of a Neutrophil Elastase Inhibitor for Interstitial Pneumonias. Ann Thorac Surg, 2017; 103: 1781-1787.

27) Hattori A, Matsunaga T, Hayashi T, Takamochi K, Oh S, Suzuki K: Prognostic Impact of the Findings on Thin-Section Computed Tomography in Patients with Subcentimeter Non-Small Cell Lung Cancer. J Thorac Oncol, 2017; 12: 954-962.

28) Aokage K, Saji H, Suzuki K, Mizutani T, Katayama H, Shibata T, Watanabe S, Asamura H; Lung Cancer Surgical Study Group of the Japan Clinical Oncology Group: A nonrandomized confirmatory trial of segmentectomy for clinical T1N0 lung cancer with dominant ground glass opacity based on thinsection computed tomography (JCOG1211). Gen Thorac Cardiovasc Surg, 2017; 65: 267272.

29) Hattori A, Suzuki K: Reply. Ann Thorac Surg, 2017; 103: 1036-1037.
30) Aokage K, Okada M, Suzuki K, Nomura S, Suzuki S, Tsubokawa N, Mimae T, Hattori A, Hishida T, Yoshida J, Tsuboi M: Is cancer history really an exclusion criterion for clinical trial of lung cancer? Influence of gastrointestinal tract cancer history on the outcomes of lung cancer surgery. Jpn J Clin Oncol, 2017; 47: 145-156.

31) Matsunaga T, Suzuki K, Takamochi K, Oh S: What is the radiological definition of partsolid tumour in lung cancer? $\dagger$ Eur J Cardiothorac Surg, 2017; 51: 242-247.

32) Hattori A, Matsunaga T, Takamochi K, Oh S, Suzuki K: Locoregional recurrence after segmentectomy for clinical-T1aNOM0 radiologically solid non-small-cell lung carcinoma. Eur J Cardiothorac Surg, 2017; 51: 518-525.

33) Yamataka A, Koga H, Ochi T, Imashimizu K, Suzuki K, Kuwatsuru R, Lane G, Nishimura K, Inada E, Suzuki K: Pulmonary lobectomy techniques in infants and children. Pediatr Surg Int, 2017; 33: 483-495.

34) Hattori A, Matsunaga T, Takamochi K, Oh S, Suzuki K: Clinical Significance of Positron Emission Tomography in Subcentimeter NonSmall Cell Lung Cancer. Ann Thorac Surg, 2017; 103: 1614-1620.

35) Kitamura Y, Suzuki K, Teramukai S, Sonobe M, Toyooka S, Nakagawa Y, Yokomise H, Date H; Japanese Association for Chest Surgery: Feasibility of Pulmonary Resection for Lung Cancer in Patients with Coronary Artery Disease or Atrial Fibrillation. Ann Thorac Surg, 2017; 103: 432-440.

36) Kawagoe I, Hayashida M, Nozumi Y, Banno T, Hirayama S, Suzuki K, Satoh D, Naito Y, Inada E: A Combination of a Partial Anomalous Pulmonary Venous Connection (PAPVC) and a Lung Tumor Requiring Pulmonary Resection. J Cardiothorac Vasc Anesth, 2017; 31: 274-278.

37) Itazawa $T$, Tamaki $Y$, Komiyama $T$, Nishimura Y, Nakayama Y, Ito $\mathrm{H}$, Ohde $\mathrm{Y}$, Kusumoto M, Sakai S, Suzuki K, Watanabe H, Asamura H: The Japan Lung Cancer SocietyJapanese Society for Radiation Oncology consensus-based computed tomographic atlas for defining regional lymph node stations in radiotherapy for lung cancer. J Radiat 
Res, 2017; 58: 86-105.

38) Tachi R, Hattori A, Matsunaga T, Takamochi K, Oh S, Suzuki K: The impact on the prognosis of unsuspected N2 disease in nonsmall-cell lung cancer: indications for thorough mediastinal staging in the modern era. Surg Today, 2017; 47: 20-26.

39) Hattori A, Matsunaga T, Takamochi K, Oh S, Suzuki K: Surgical Management of Multifocal Ground-Glass Opacities of the Lung: Correlation of Clinicopathologic and Radiologic Findings. Thorac Cardiovasc Surg, 2017; 65: 142-149.

40) Hattori A, Suzuki K: Do Tumor Size and Carcinoembryonic Antigen Level Affect Surgical Management of Partially Solid EarlyStage Lung Cancer? Reply. Ann Thorac Surg, 2017; 103: 1036-1037.

41) Fukui M, Takamochi K, Oh S, Matsunaga T, Suzuki K, Ando K, Suzuki K: A Prospective Randomized Trial of Perioperative Administration of Neutrophil Elastase Inhibitor in Patients with Interstitial Pneumonias. J Thorac Oncol, 2017; 12: S1401.

42) Hata K, Suzuki K, Matsunaga T, Takamochi K, Oh S: The Feasibility of Lung Second Surgery for 2nd Primary Lung Cancer. J Thorac Oncol, 2017; 12: S772-S773.

43) Hattori A, Matsunaga T, Takamochi K, Oh S, Suzuki K: Proposals for the Novel Clinical T Categories Based on the Presence of Ground Glass Opacity Component in Lung Adenocarcinoma. J Thorac Oncol, 2017; 12: S399-S400.

44) Hirayama S, Matsunaga $T$, Takamochi K, Oh S, Suzuki K: Perioperative Management of Antiplatelet Therapy in Patients with Coronary Stent Who Need Thoracic Surgery. J Thorac Oncol, 2017; 12: S1398.

45) Koike Y, Hattori A, Matsunaga T, Takamochi K, Oh S, Suzuki K: Feasibility of Lung Cancer Surgery for the Patient with Previous History of Coronary Artery Bypass Grafting. J Thorac Oncol, 2017; 12: S1400.

46) Kunitoh H, Sakurai H, Tsuboi M, Wakabayashi M, Okada M, Suzuki K, Ikeda N, Takenoyama M, Ohde Y, Takahama M, Yoshiya K, Matsumoto I, Yamashita M, Marutsuka T, Date H, Saito Y, Yamashita Y, Okumura N, Watanabe S, Asamura H: Safety and Compliance Data of the Phase III Study of Adjuvant Chemotherapy in Completely Resected P-Stage I Non-Small Cell Lung Cancer: JCOG0707. J Thorac Oncol, 2017; 12: S646.

47) Oh S, Takamochi K, Suzuki K: Retrospective Study of Pleuropneumonectomy for Thymoma with Dissemination. J Thorac Oncol, 2017; 12: S1005.

48) Ouchi T, Hattori A, Matsunaga T, Takamochi K, Oh S, Suzuki K: Feasibility of Surgical Resection for Lung Cancer Patients Aged over 85 Years. J Thorac Oncol, 2017; 12: S736S737.

49) Suzuki J, Hattori A, Takamochi K, Oh S Suzuki K: Ground-Glass Opacity (GGO) with Semi-Consolidation: Clinicopathological and Radiological Correlations Compared to PureGGOs of the Lung. J Thorac Oncol, 2017; 12: S555-S556.

50) Suzuki M, Matsunaga T, Takamochi K, Oh Si, Suzuki K: Impact of Visceral Plural Invasion to T Descriptors: Based on the Forthcoming Eighth Edition of TNM Classification for Lung Cancer. J Thorac Oncol, 2017; 12: S588-S588.

51) Takamochi K, Oh S, Matsunaga T, Suzuki K: The Prognostic Impact of EGFR Mutation Status and Mutation Subtypes in Patients with Surgically Resected Lung Adenocarcinomas. J Thorac Oncol, 2017; 12: S613-S614.

52) Sato M, Kuwata T, Yamanashi K, Kitamura A, Misawa K, Imashimizu K, Kobayashi M, Ikeda M, Koike T, Kosaka S, Fukai R, Sekine Y, Isowa N, Hirayama S, Sakai H, Watanabe F, Nagayama K, Aoyama A, Date H, Nakajima J: Safety and reproducibility of virtual-assisted lung mapping: a multicentre study in Japan. Eur J Cardiothorac Surg, 2017; 51: 861-868.

53) Hattori A, Matsunaga T, Takamochi K, Oh S, Suzuki K: Importance of ground glass opacity component in clinical stage IA radiological invasive lung cancer. Ann Thorac Surg, 2017; 104: 313-320.

54) Hattori A, Matsunaga T, Hayashi T, Takamochi K, Oh S, Suzuki K: Prognostic impact of the findings on thin-section computed tomography in patients with subcentimeter non-small cell lung cancer. J Thorac Oncol, 2017; 12: 954-962. 
55) Hattori A, Matsunaga T, Takamochi K, Oh S, Suzuki K: Indication for sublobar resection of clinical-stage IA radiological pure-solid lung adenocarcinoma. J Thorac Cardiovasc Surg, 2017; 154: 1100-1108.

56) Hattori A, Matsunaga T, Takamochi K, Oh S, Suzuki K: Clinical significance of positron emission tomography in subcentimeter nonsmall cell lung cancer. Ann Thorac Surg, 2017; 103: 1614-1620.

57) Hattori A, Matsunaga T, Takamochi K, Oh S, Suzuki K: Prognostic impact of a ground glass opacity component in the clinical $\mathrm{T}$ classification of non-small cell lung cancer. J Thorac Cardiovasc Surg, 2017; 154: 21022110.e1.

58) Hattori A, Matsunaga T, Takamochi K, Oh S, Suzuki K: Radiological classification of multiple lung cancers and the prognostic impact based on the presence of a ground glass opacity component on thinsection computed tomography. Lung Cancer, 2017; 113: 7-13.

59) Hattori A, Suzuki K: Controversies of the optimal surgical management for the lymph nodes in non-small cell lung cancer. Shanghai Chest, 2017; 1: 38.

〈Reviews〉

1) Aokage K, Yoshida J, Hishida T, Tsuboi M, Saji H, Okada M, Suzuki K, Watanabe S, Asamura H: Limited resection for earlystage non-small cell lung cancer as functionpreserving radical surgery: a review. Jpn J Clin Oncol, 2017; 47: 7-11.

\section{Department of Pediatric General and Urogenital Surgery}

\section{$\langle$ Original Articles〉}

1) Yamataka A, Koga H, Ochi T, Imashimizu K, Suzuki K, Kuwatsuru R, Lane G, Nishimura K, Inada E, Suzuki K: Pulmonary lobectomy techniques in infants and children. Pediatr Surg Int, 2017; 33: 483-495.

2) Yamataka A, Miayno G, Takeda M: Minimally invasive neonatal sugery: Hirschsprung disease. Clin Perinatol, 2017; 44: 851864.

3) Koga H, Okawada M, Miyano G, Doi T, Lane GJ, Yamataka A: Is measuring the residual rectourethral fistula during laparoscopically assisted anorectal pull-through for male high/intermediate type imperforate anus beneficial? Mid-term follow-up. J Pediatr Surg, 2017; 52: 1994-1996.

4) Cazares J, Koga H, Murakami H, Nakamura H, Lane GJ, Yamataka A: Laparoscopic portoenterostomy for biliary atresia: singlecenter experience and review of literatures. Pediatr Surg Int, 2017; 33: 1341-1354.

5) Miyano G, Seo S, Nakamura H, Sueyoshi R, Okawada M, Doi T, Koga H, Lane GJ, Yamataka A: Changes in quality of life from infancy to school age after esophagoesophagostomy for tracheoesophageal fistula: thoracotomy versus thoracoscopy. Pediatr Surg Int, 2017; 33: 1087-1090.

6) Miyano G, Nakamura H, Seo S, Sueyoshi R, Okawada M, Doi T, Koga H, Lane GJ, Okazaki T, Yamataka A: Laparoscopy-assisted Duhamel- $Z$ anastomosis for total colonic aganglionosis: outcome assessed by fecal continence evaluation. J Laparoendosc Adv Surg Tech A, 2017; 27: 302-305.

7) Miyano G, Koyama M, Miyake H, Kaneshiro M, Morita K, Nakajima H, Yamoto M, Nouso H, Fukumoto K, Urushihara N: Comparison of laparoscopic hepaticojejunostomy and open hepaticojejunostomy. Can stenosis of the hilar hepatic duct affect postoperative outcome? Asian J Endosc Surg, 2017; 10: 295-300.

8) Okazaki T, Okawada M, Ishii J, Koga H, Miyano G, Doi T, Ogasawara Y, Lane GJ, Yamataka A: Intraoperative ventilation during thoracoscopic repair of neonatal congenital diaphragmatic hernia. Pediatr Surg Int, 2017; 33: 1097-1101.

9) Okazaki T, Yamataka A, Asahara T, Nomoto $\mathrm{K}$, Yamashiro Y: The high incidence of bacteremia in children undergoing surgery can be prevented by Bifidobacterium supplementation. Ann Nutr Metab, 2017; 71 (Suppl 1): 31-36.

10) Okazaki T, Okawada M, Yamataka A: Reply to letter to the editor concerning- "Congenital diaphragmatic hernia in neonates: factors related to failure of thoracoscopic repair". Pediatr Surg Int, 2017; 33: 515.

11) Takahashi T, Friedmacher F, Zimmer J, Puri 
P: N-deacetylase-N-sulfotransferase-1 signaling in the developing diaphragmatic vasculature of nitrofen-induced congenital diaphragmatic hernia. J Pediatr Surg, 2017; 52: 1035-1039.

12) Takahashi T, Friedmacher F, Zimmer J, Puri P: Follistatin-like 1 expression is decreased in the alveolar epithelium of hypoplastic rat lungs with nitrofen-induced congenital diaphragmatic hernia. J Pediatr Surg, 2017; 52: 706-709.

13) Takahashi $T$, Friedmacher F, Zimmer J, Puri $\mathrm{P}$ : Expression of T-box transcription factors 2, 4 and 5 is decreased in the branching airway mesenchyme of nitrofen-induced hypoplastic lungs. Pediatr Surg Int, 2017; 33: 139-143.

14) Takahashi $T$, Friedmacher F, Zimmer J, Puri P: Fibrillin-1 expression is decreased in the diaphragmatic muscle connective tissue of nitrofen-induced congenital diaphragmatic hernia. Eur J Pediatr Surg, 2017; 27: 26-31.

15) Nakamura H, Henderson D, Puri P: A metaanalysis of clinical outcome of intestinal transplantation in patients with total intestinal aganglionosis. Pediatr Surg Int, 2017; 33: 837-841.

16) Nakamura H, Koga H, Miyano G, Okawada M, Doi T, Yamataka A: Does the Level of Transection of the Biliary Remnant Affect Outcome After Laparoscopic Kasai Portoenterostomy for Biliary Atresia? Pediatr Surg Int, 2017; 33: 837-841.

17) Seo S, Miyake H, Koike Y, Chen Y, Hock A, Lee C, Li B, Perro A: Duhamel and Transanal Endorectal pull-throughs for Hirschsprung's Disease: A Systematic Review and MetaAnalysis. Eur J Pediatr Surg, Epub 2017 Sep 28.

18) Takeda M, Miyahara K, Akazawa C, Lane GJ, Yamataka A: Sensory innervation of the anal canal and anorectal line in Hirschsprung's disease: histological evidence from mouse models. Pediatr Surg Int, 2017; 33: 883-886.

〈Books〉

1) Koga H, Yamataka A: Chapter 76. Congenital biliary dilatation. In: Puri P, ed. Newborn surgery 4th edn. London; Taylor \& Francis Group, 2017.
2) Okawada M, Lane GJ, Yamataka A: Part 1 General-6, Bone Mineral Density as a Marker for Pectus Correction. In: Saxena AK, ed. Chest Wall Deformities. Switzerland; Springer, 2017; 75-79.

\section{Department of Urology}

〈Original Articles〉

1) Kanayama M, Hayano T, Koebis M, Maeda T, Tabe Y, Horie S, Aiba A: Hyperactive mTOR Induces Neuroendocrine Differentiation in Prostate Cancer Cell with Concurrent Upregulation of IRF1. Prostate, 2017; 77: 14891498.

2) Luo J, Attard G, Balk SP, Bevan C, Burnstein K, Cato L, Cherkasov A, De Bono JS, Dong Y, Gao AC, Gleave M, Heemers H, Kanayama M, Kittler R, Lang JM, Lee RJ, Logothetis CJ, Matusik R, Plymate S, Sawyers CL, Selth LA, Soule H, Tilley W, Weigel NL, Zoubeidi A, Dehm SM, Raj GV: Role of androgen receptor variants in prostate cancer: report from the 2017 Mission Androgen Receptor Variants meeting. Eur Urol, Epub 2017 Dec 16.

3) Muto S, Okada T, Yasuda M, Tsubouchi H, Nakajima K, Horie S: Long-term safety profile of tolvaptan in autosomal dominant polycystic kidney disease patients: TEMPO Extension Japan Trial. Drug Healthc Patient Saf, 2017; 9: 93-104.

4) Muto S, Kitamura K, Ieda T, Shimizu F, Nagata M, Isotani S, Ide H, Yamaguchi R, Horie S: A preliminary oncologic outcome and postoperative complications in patients undergoing robot-assisted radical cystectomy: Initial experience. Investig Clin Urol, 2017; 58: 171-178.

5) Ide H, Lu Y, Yu J, Noguchi T, Kanayama M, Muto S, Yamaguchi R, Kawato S, Horie S: Aryl hydrocarbon receptor signaling involved in the invasiveness of LNCaP cells. Hum Cell, 2017; 30: 133-139.

6) Shimoyama H, Isotani S, China T, Nagata M, Yokota I, Kitamura K, Wakumoto Y, Ide H, Muto S, Tujimura A, Yamaguchi R, Horie S: Automated renal cortical volume measurement for assessment of renal function in patients undergoing radical nephrectomy. Clin Exp Nephrol, 2017; 21: 1124-1130. 
7) Shimizu F, Muto S, Taguri M, Ieda $T$, Tsujimura A, Sakamoto Y, Fujita K, Okegawa T, Yamaguchi R, Horie S: Effectiveness of platinum-based adjuvant chemotherapy for muscle-invasive bladder cancer, a weighted propensity score analysis. Int $\mathrm{J}$ Urol, 2017; 24: 367-372.

8) Uchida T, Nishimoto K, Fukumura Y, Asahina M, Goto H, Kawano Y, Shimizu F, Tsujimura A, Seki T, Mukai K, Kabe Y, Suematsu M, Gomez-Sanchez CE, Yao T, Horie S, Watada H: Disorganized steroidgenesis in adrenocortical carcinoma, a case study. Endocr Pathol, 2017; 28: 27-35.

9) Shimizu F, Muto S, Taguri M, Ieda $T$, Tsujimura A, Sakamoto Y, Fujita K, Okegawa T, Yamaguchi R, Horie S: Effectiveness of platinum-based adjuvant chemotherapy for muscle-invasive bladder cancer: A weighted propensity score analysis. Int J Urol, 2017; 24: 367-372.

10) Tatsuzawa $M$, Ogawa $R$, Kinjo $N$, Kim $S$, Shimizu F, Sakamoto Y, Shimojima K, Echizen H, Miyazaki A: Consequences of different corticosteroids on serum potassium and prostate-specific antigen in patients receiving abiraterone for astration-resistant prostate cancer: A retrospective observational study. Clin Med Insights Oncol, 2017; 11: 1-7.

11) Mizuno T, Hiramatsu I, Aoki $Y$, Shimoyama H, Nozaki T, Shirai M, Lu Y, Horie S, Tsujimura A: Relation between histological prostatitis and lower urinary tract symptoms and erectile function. Prostate Int, 2017; 5: 119-123.

12) Tsujimura A, Hiramatsu I, Aoki $Y$, Shimoyama H, Mizuno T, Nozaki T, Shirai M, Kobayashi K, Kumamoto Y, Horie S: Atherosclerosis is associated with erectile function and lower urinary tract symptoms, especially nocturia, in middle-aged men. Prostate Int, 2017; 5: 65-69.

13) Tsujimura A, Kiuchi $H$, Soda $T$, Takezawa K, Fukuhara S, Takao T, Sekiguchi Y, Iwasa A, Nonomura N, Miyagawa Y: The pattern of sexual interest to female-to-male transsexual persons with gender identity disorder dose not resemble that of biological men: an eye- tracking study. Sex Med, 2017; 5: e169-e174.

14) Sato $Y$, Tajima A, Katsurayama M, Nozawa S, Yoshiike M, Koh E, Kanaya J, Namiki M, Matsumiya K, Tsujimura A, Komatsu K, Itoh N, Eguchi J, Imoto I, Yamauchi A, Iwamoto T: An independent validation study of three single nucleotide polymorphisms at the sex hormone-binding globulin locus for testosterone levels identified by genome-wide association studies. Hum Reprod Open, 2017; 2017: hox002.

15) Soda T, Miyagawa Y, Ueda N, Takezawa K, Okuda H, Fukuhara S, Fujita K, Kiuchi H, Uemura M, Okamoto Y, Tsujimura A, Tanaka H, Nonomura N: Systematic characterization of human testis-specific actin capping protein $\beta 3$ as a possible biomarker for male infertility. Hum Reprod 2017; 32: 514522.

16) Shimoyama H, Isotani S, China T, Nagata $M$, Yokota I, Kitamura K, Wakumoto Y, Ide H, Muto, Tsujimura A, Yamaguchi R, Horie S: Renal cortical volume measurement of renal function in patient undergoing radical nephrectomy. Clin Exp Nephrol, 2017; 6: 11241130.

17) Mizuno T, Hiramatsu I, Aoki $Y$, Shimoyama H, Nozaki T, Shirai M, Lu Y, Horie S, Tsujimura A: Relation between histological prostatitis and lower urinary tract symptom as well as erectile function. Prostate Int, 2017; 5: $119-123$.

18) Shirai M, Tsujimura A, Abdelhamed A, Horie S: Sexuality education in Japanese medical school. Int J Imp Res, 2017; 29: 160-163.

19) Yumura $Y$, Tsujimura A, Imamoto $T$, Umemoto Y, Kobayashi H, Shiraishi K, Shin T, Taniguchi H, Chiba K, Miyagawa Y, Iwamoto T: Nationwide survey of urological specialists regarding male infertility: results from a 2015 questionnaire in Japan. Reprod Med Biol, 2017; 17: 44-51.

20) Shirai M, Tsujimura A, Abdelhamed A, Horie S: Sexuality education in Japanese medical schools. Int J Impot Res, 2017; 29: 160-163.

21) Horie S: [The Aim and Scope of "Clinical Practice Guidelines for the Management of Kidney Disease in Cancer Survivors"]. Gan To Kagaku Ryoho, 2017; 44: 191-195. 
22) Kugita M, Nishii K, Yamaguchi T, Suzuki A, Yuzawa Y, Horie S, Higashihara E, Nagao S: Beneficial effect of combined treatment with octreotide and pasireotide in PCK rats, an orthologous model of human autosomal recessive polycystic kidney disease. PLoS One, 2017; 12: e0177934.

23) Mizuno R, Kimura G, Fukasawa S, Ueda T, Kondo T, Hara H, Shoji S, Kanao K, Nakazawa H, Tanabe K, Horie S, Oya M; Tokyo Metropolitan Study Group: Angiogenic, inflammatory and immunologic markers in predicting response to sunitinib in metastatic renal cell carcinoma. Cancer Sci, 2017; 108: 1858-1863.

24) Kim CS, Lee JY, Chung BH, Kim WJ, Fai NC, Hakim L, Umbas R, Ong TA, Lim J, Letran JL, Chiong E, Wu TL, Lojanapiwat B, Türkeri L, Murphy DG, Gardiner RA, Moretti K, Cooperberg M, Carroll P, Mun SK, Hinotsu S, Hirao Y, Ozono S, Horie S, Onozawa M, Kitagawa Y, Kitamura T, Namiki M, Akaza H: Report of the Second Asian Prostate Cancer (A-CaP) Study Meeting. Prostate Int, 2017; 5: 95-103.

25) Horie S: Sensing the texture of imaging, a new biomarker of polycystic kidney disease. Kidney Int, 2017; 92: 1044-1045.

$\langle$ Reviews〉

1) Nagata M, Horie S: Diagnosis and treatment in the era of precision medicine - Precision medicine and tailor-made medicine. Personalized Med Universe, 2017; 6: 1-3.

\section{Department of Transfusion Medicine \& Stem Cell Regulation}

$\langle$ Original Articles〉

1) Kitajima S, Lee KL, Hikasa H, Sun W, Huang RY, Yang H, Matsunaga S, Yamaguchi T, Araki M, Kato H, Poellinger L: Hypoxiainducible factor-1alpha promotes cell survival during ammonia stress response in ovarian cancer stem-like cells. Oncotarget, 2017; 8: 114481-114494.

* 2) Misawa K, Yasuda H, Araki M, Ochiai T, Morishita S, Nudejima M, Hironaka Y, Shirane S, Edahiro Y, Gotoh A, Ohsaka A,
Komatsu N: The 2016 WHO diagnostic criteria for polycythemia vera renders an accurate diagnosis to a broader range of patients including masked polycythemia vera: Comparison with the 2008 WHO diagnostic criteria. Am J Hematol, 2017; 92: E128-E130.

3) Sunami Y, Araki M, Kan S, Ito A, Hironaka Y, Imai M, Morishita S, Ohsaka A, Komatsu N: Histone Acetyltransferase p300/CREB-binding Protein-associated Factor (PCAF) Is Required for All-trans-retinoic Acidinduced Granulocytic Differentiation in Leukemia Cells. J Biol Chem, 2017; 292: 28152829.

4) Ai T, Yuri M, Tabe Y, Kakimoto A, Morishita S, Tsuchiya K, Takamochi K, Kodama Y, Takahashi F, Shigeki M, Horii T, Suzuki K, Takahashi K, Miida T, Ohsaka A: Comparison of the Analytical Performance Between cobas EGFR Assay and PCR-Clamp Method in the Detection of EGFR Mutations in Japanese Non-Small Cell Lung Cancer Patients. Clin Lab, 2017; 63: 1021-1026.

5) Ueda K, Ikeda K, Ikezoe T, Harada-Shirado K, Ogawa K, Hashimoto Y, Sano T, Ohkawara H, Kimura S, Shichishima-Nakamura A, Nakamura Y, Shikama Y, Mori T, Mason PJ, Bessler M, Morishita S, Komatsu N, Shide K, Shimoda K, Koide S, Aoyama K, Oshima M, Iwama A, Takeishi Y: Hmga2 collaborates with JAK2V617F in the development of myeloproliferative neoplasms. Blood Adv, 2017; 1: 1001-1015.

〈Reviews〉

1) Imai $M$, Araki $M$, Komatsu N: Somatic mutations of calreticulin in myeloproliferative neoplasms. Int J Hematol, 2017; 105: 743-747.

2) Araki M, Komatsu N: Novel molecular mechanism of cellular transformation by a mutant molecular chaperone in myeloproliferative neoplasms. Cancer Sci, 2017; 108: 1907-1912.

3) Ohsaka A: Electronic pre-transfusion check at the bedside: experience in a university hospital. Hematol Transfus Int J, 2017; 4: 90-96. 